\title{
Equilibrium and observational properties of line-tied twisted flux tubes
}

\author{
G. Aulanier ${ }^{1}$, P. Démoulin ${ }^{1}$, and R. Grappin ${ }^{2}$ \\ 1 Observatoire de Paris, LESIA, 92195 Meudon Cedex, France \\ e-mail: [guillaume.aulanier; pascal .demoulin]@obspm.fr \\ 2 Observatoire de Paris, LUTH, 92195 Meudon Cedex, France \\ e-mail: roland.grappin@obspm.fr
}

Received 23 June 2004 / Accepted 19 September 2004

\begin{abstract}
We describe a new explicit three-dimensional magnetohydrodymanic code, which solves the standard zero- $\beta$ MHD equations in Cartesian geometry, with line-tied conditions at the lower boundary and open conditions at the other ones. Using this code in the frame of solar active regions, we simulate the evolution of an initially potential and concentrated bipolar magnetic field, subject to various sub-Alfvénic photospheric twisting motions which preserve the initial photospheric vertical magnetic field. Both continuously driven and relaxation runs are performed. Within the numerical domain, a steep equilibrium curve is found for the altitude of the apex of the field line rooted in the vortex centers as a function of the twist. Its steepness strongly depends on the degree of twist in outer field lines rooted in weak field regions. This curve fits the analytical expression for the asymptotic behaviour of force-free fields of spherical axisymmetric dipoles subject to azimuthal shearing motions, as well as the curve derived for other line-tied twisted flux tubes reported in previous works. This suggests that it is a generic property of line-tied sheared/twisted arcades. However, contrary to other studies we never find a transition toward a non-equilibrium within the numerical domain, even for twists corresponding to steep regions of the equilibrium curve. The calculated configurations are analyzed in the frame of solar observations. We discuss which specific conditions are required for the steepness of the generic equilibrium curve to result in dynamics which are typical of both fast and slow CMEs observed below $3 R_{\odot}$. We provide natural interpretations for the existence of asymmetric and multiple concentrations of electric currents in homogeneoulsy twisted sunspots, due to the twisting of both short and long field lines. X-ray sigmoïds are reproduced by integrating the Joule heating term along the line-of-sight. These sigmoïds have inverse-S shapes associated with negative force-free parameters $\alpha$, which is consistent with observed rules in the northern solar hemisphere. We show that our sigmoïds are not formed in the main twisted flux tube, but rather in an ensemble of low-lying sheared and weakly twisted field lines, which individually never trace the whole sigmoïd, and which barely show their distorded shapes when viewed in projection. We find that, for a given bipolar configuration and a given twist, neither the $\alpha$ nor the altitude of the lines whose envelope is a sigmoïd depends on the vortex size.
\end{abstract}

Key words. magnetohydrodynamics (MHD) - methods: numerical - Sun: magnetic fields - Sun: photosphere - Sun: corona

\section{Introduction}

Several astrophysical objects are composed of an extended diluted medium filled by highly ionized plasma, which is coupled to a much denser region by magnetic fields which pass from one medium to the other. Some stellar atmospheres, and the environment of hot accretion disks, fall into this class. The case of the Sun is particularly interesting in the study of such couplings, since its proximity provides detailed observations which put strong constraints on the theory. Below helmet streamers the low solar corona is composed of a collisional plasma. Its $\beta$ parameter is well below unity, so that it is dominated by magnetic fields. Its pressure scale-height is of the order of the largest observable structures, and its Lundquist number is larger than one by orders of magnitude. The coronal magnetic fields are not "independent", since they penetrate the dense solar interior through the photospheric interface in sunspot and network regions, where $\beta$ and the pressure scale height increase rapidly with depth as the photosphere becomes opaque. The density gradients then imply that magnetosonic waves emitted in the corona are almost fully reflected by the photosphere. So the photospheric dynamics (whose time-scales are typically much larger than the Alfvén ones) are barely influenced by the coronal evolution. However, the long-term evolution of the corona is dominated by the photospheric forcing. In this context, the evolution of coronal magnetic fields forced by slow photospheric motions can be studied in the frame of magnetohydrodynamics (MHD).

The very different physical regimes in the two media are very difficult to treat numerically altogether, so that two 
major simplications can be made. Firstly, the photosphere can be treated as a so-called "line-tied" boundary. This extreme assumption implies that this boundary is infinitely conductive, inertial and reflective. Secondly, the low corona can be treated as a pressureless medium $(\beta=0)$ in which gravity plays no role, so that only the Lorentz force can accelerate the plasma. Even though this last assumption is not always made, it becomes mandatory so as to treat large systems having magnetic field contrasts of several orders of magnitude, so as to limit the Alfvén speed variations. In this paper we perform a limited parametric study of line-tied bipolar flux tubes twisted by two simple photospheric vortices, and we compare our results with those previously published on the same topic. The aim is to derive generic properties of such systems.

Since magnetic extrapolations (e.g. Schmieder et al. 1996) and vector magnetograms (e.g. Leka \& Skumanich 1999) have shown that the magnetic fields are often non-potential (i.e. that distributed electric currents exist) in the solar atmosphere, three-dimensional sheared and twisted line-tied flux tubes have already been modeled, either starting from uniform or from potential fields. Note that even though some rotating sunspots have been reported in observations (e.g. Brown et al. 2003) and references therein), the shearing/twisting motions in MHD simulations, including ours, have always been ad-hoc and almost never pretended to simulate true photospheric velocity fields. They are considered either on a purely theoretical basis, or so as to simulate approximately the apparent photospheric displacement of field line footpoints induced by the emergence through the photosphere of flux tubes which have previously been twisted in the solar interior, as observed e.g. by Leka et al. (1996).

All theoretical studies have shown that for moderate footpoint displacements, the system can always find an equilibrium (being force-free in the zero- $\beta$ case). However, the behaviour of the system appears to be qualitiatively different depending on the geometry. In a cylindrical geometry invariant by rotation with two line-tied end-plates, flux tubes subject to different classes of twisting motions always become unstable to the kink mode, typically when the increasing toroidal component of the magnetic field becomes comparable to the axial field (see e.g. Mikic et al. 1990; Baty 2000, 2001; Hood 1992, for a review). This instability subsequently results in the formation of thin current sheets, in which magnetic reconnexion occurs (see e.g. Baty 2001). In an axisymmetric spherical geometry, sheared flux surfaces exhibit a highly increasing expansion rate as a function of shear, but always following a sequence of equilibrium states in ideal MHD (Roumeliotis et al. 1994; Mikic \& Linker 1994). Note that Sturrock et al. (1995) derived an analytical expression for the asymptotic behaviour of these equilibria as a function of footpoint displacement (see our Eq. (42)). In this geometry, the only known mechanisms to achieve a loss of equilibrium are resistive effects, either within highly sheared arcades (Mikic \& Linker 1994) or within surrounding separatrices formed in quadrupolar topologies (Antiochos et al. 1999). In Cartesian geometry, very few calculations of simple twisting of initial potential fields have been performed without introducing special boundary effects so as to create flux ropes and ejections. Using very concentrated vortices embedded in strong fields, and reaching an end-to-end twist of about 1.4 turns, van Hoven et al. (1995) always found an equilibrium. Considering a similar system, Amari \& Luciani (1999) on the contrary found a kink-like instability which resulted in a resistive disruption of the system associated with magnetic reconnection between twisted and overlaying potential fields. In the same context, but using a fixed uniform density (which leads to a sharp decrease of Alfvén speed with altitude), Tokman \& Bellan (2002) calculated that highly twisted configurations exhibit some dynamic behaviour after magnetic reconnection occurs at several locations within the twisted flux tube. Neither calculations from Amari \& Luciani (1999) nor from Tokman \& Bellan (2002) resulted in a strong vertical expansion of the system during their respective dynamic phases. Using more extended vortices and a non-uniform density distribution, Amari et al. (1996) first revealed the "very fast opening" of a continuously twisted system after some critical twist was reached. Klimchuk et al. (2000) calculated non-linear force-free field models of a similar configuration up to one turn, noting that numerical relaxations became increasingly long at the twist increased. Very recently, Török \& Kliem (2003) calculated several systems comparable to the one studied in Amari et al. (1996). They showed that their equilibrium curves were very steep, and that they surprisingly followed the Sturrock et al. (1995) expression. Performing a more detailed analysis of one of their systems, they found a loss of equilibrium for twists larger than 1.38-1.48 turns, characterized by a fast vertical expansion of the mostly twisted flux. By analogy with calculations in cylindrical geometry, they attributed this behaviour to a kink-like instability. This might also be responsible for the "very fast opening" in Amari et al. (1996), as proposed in Baty (2000). In spite of all these studies, no firm generic explanation has yet been firmly advanced for the change of behaviour of twisted flux tubes for twists larger than $1+\epsilon$ turns in Cartesian geometry: Is it really due to a kink instability as in the cylindrical case, or is it due to an increasing expansion rate of equilibrium states as in the axisymmetric case? Answering this question requires a parametric study using fully time-dependant MHD equations. It is the first objective of the present paper.

Apart from theoretical considerations, it would be insteresting to compare typical properties of line-tied twisted flux tubes with typical solar observations. This would not only permit to test the applicability of such calculations to the real Sun, but perhaps help to understand complex observations with simple (and controllable) physical effects. Unfortunately, apart from the brief mention of S-shape field lines and/or electric currents by Amari et al. (1996) and Török \& Kliem (2003), no detailed analysis was ever published according to the author's knowledge. In this paper we address three fundamental issues which result from typical observations of the solar photosphere and corona.

Firstly, what is the origin of the typical fragmentation (often accompanied by changes in sign) of electric currents calculated from vector magnetograms observed in sunspots (see e.g. Pevtsov et al. 1995; Leka \& Skumanich 1999; Régnier et al. 2002)? Secondly, can current-carrying magnetic field lines fully trace sigmoïdal structures often observed in X-rays in the corona projected onto the solar disc 
(Manoharan et al. 1996; Rust \& Kumar 1996; Sterling \& Hudson 1997; Hudson et al. 1998; Pevtsov \& Canfield 1999; Gibson et al. 2002), as they typically do for other X-ray and EUV loops (see e.g. Schmieder et al. 1996; Démoulin et al. 2002; Burnette et al. 2004)? In other words, are sigmoïds a quantitative tracer of magnetic twist? Also, why are sigmoïds barely observed at the limb, and what is their typical altitude? Thirdly, can the dynamics of simple bipolar twisted flux tubes result in the typical height-time plots observed in coronal mass ejections (CMEs) at low altitude below helmet streamers (Srivastava et al. 1999, 2000; Wang et al. 2003; Zhang et al. 2004)? Also, can a simple explanation be found for the existence of two classes of CMEs (Gosling et al. 1976; MacQueen \& Fisher 1983; Delannée et al. 2000; Andrews \& Howard 2001 ), i.e. the fast/flare-related ones and the slow/prominencerelated others?

The plan of the paper is the following: in Sect. 2 we describe the main features of the code. The initial settings of the calculations are given in Sect. 3. In Sect. 4 we analyze the evolution of the magnetic configuration while it is continuously driven at the line-tied boundary. In Sect. 5 we report on the equilibrium analysis of the system for various parameters, and we numerically construct equilibrium curves for which we provide analytical expressions. In Sect. 6 we analyze the calculations in the frame of solar observations of photospheric electric currents and of X-ray sigmoïds. We also discuss the possible applications as well as the limitations of these calculations for coronal mass ejections. The results are summarized in Sect. 7.

\section{General description of the MHD code}

Since this paper reports on the first application of our new code, this section explains in detail the numerical method.

\subsection{Set of equations}

The standard zero- $\beta$ (pressureless) time-dependant MHD equations for a fully ionised and collisional plasma can be written as:

$\frac{\partial \rho}{\partial t}=-\nabla \cdot(\rho \boldsymbol{u})$

$\rho \frac{\partial \boldsymbol{u}}{\partial t}=-\rho(\boldsymbol{u} \cdot \boldsymbol{\nabla}) \boldsymbol{u}+\boldsymbol{J} \times \boldsymbol{b}+\rho \mathfrak{D} \boldsymbol{u}$

$\frac{\partial \boldsymbol{b}}{\partial t}=\boldsymbol{\nabla} \times(\boldsymbol{u} \times \boldsymbol{b})+\mathfrak{R} \boldsymbol{b}$

$\boldsymbol{\nabla} \times \boldsymbol{b}=\mu \boldsymbol{J}$

$\boldsymbol{\nabla} \cdot \boldsymbol{b}=0$

where $\rho$ is the mass density, $\boldsymbol{u}$ is the plasma velocity, $\boldsymbol{b}$ is the magnetic field, $\boldsymbol{J}$ is the electric current density and $\mu$ is the magnetic permeability. $\mathfrak{D}$ and $\mathfrak{R}$ are respectively the diffusion operators for the velocity and the magnetic field, which will be defined below.

Our code solves these equations in three dimensions in Cartesian geometry $(x ; y ; z)$, in their fully developed form.
Using Einstein's notation for spatial derivatives (where subscripts $i ; j$ can be $x ; y ; z)$, these equations are written in our code as:

$$
\begin{aligned}
\partial_{t} \rho= & -u_{j} \partial_{j} \rho-\rho \partial_{j} u_{j} \\
\partial_{t} u_{x}= & +(\mu \rho)^{-1}\left(b_{y} \partial_{y} b_{x}-b_{y} \partial_{x} b_{y}+b_{z} \partial_{z} b_{x}-b_{z} \partial_{x} b_{z}\right) \\
& -u_{j} \partial_{j} u_{x}+\mathfrak{D} u_{x} \\
\partial_{t} u_{y}= & +(\mu \rho)^{-1}\left(b_{z} \partial_{z} b_{y}-b_{z} \partial_{y} b_{z}+b_{x} \partial_{x} b_{y}-b_{x} \partial_{y} b_{x}\right) \\
& -u_{j} \partial_{j} u_{y}+\mathfrak{D} u_{y} \\
\partial_{t} u_{z}= & +(\mu \rho)^{-1}\left(b_{x} \partial_{x} b_{z}-b_{x} \partial_{z} b_{x}+b_{y} \partial_{y} b_{z}-b_{y} \partial_{z} b_{y}\right) \\
& -u_{j} \partial_{j} u_{z}+\mathfrak{D} u_{z} \\
\partial_{t} b_{x}= & -u_{j} \partial_{j} b_{x}-b_{x} \partial_{j} u_{j}+b_{j} \partial_{j} u_{x}+\Re b_{x} \\
\partial_{t} b_{y}= & -u_{j} \partial_{j} b_{y}-b_{y} \partial_{j} u_{j}+b_{j} \partial_{j} u_{y}+\Re b_{y} \\
\partial_{t} b_{z}= & -u_{j} \partial_{j} b_{z}-b_{z} \partial_{j} u_{j}+b_{j} \partial_{j} u_{z}+\Re b_{z} .
\end{aligned}
$$

These equations are solved on a discretized fixed mesh, having $\left(n_{x} ; n_{y} ; n_{z}\right)$ points which can be prescribed non-uniformly in the $(x ; y ; z)$ directions. All quantities (including their spatial derivatives) are specified at the same locations, i.e. gridpoints.

In the following, we define $\mathrm{d} x^{i}$ (resp. $\mathrm{d} y^{i} ; \mathrm{d} k^{i}$ ) as the distance between the $i$ th and $i+1$ th gridpoints, and $\mathrm{d}^{\prime} x^{i}$ (resp. $\mathrm{d}^{\prime} y^{i} ; \mathrm{d}^{\prime} z^{i}$ ) as the minimal distance between the $i$ th gridpoint and its two neighbors, along the $x$ (resp. $y ; z$ ) axis:

$\mathrm{d} x^{i}=x^{i+1}-x^{i}$

$\mathrm{d}^{\prime} x^{i}=\min \left(\mathrm{d} x^{i} ; \mathrm{d} x^{i-1}\right)$.

Then we define $\mathrm{d}^{i, j, k}$ as the smallest length-scale available at a given position, and $d$ as the smallest length-scale available in a given mesh:

$\mathrm{d}^{i, j, k}=\min \left(\mathrm{d}^{\prime} x^{i} ; \mathrm{d}^{\prime} y^{j} ; \mathrm{d}^{\prime} z^{k}\right)$

$d=\min \left(d^{i, j, k}\right)$.

Equation (15) permits us to define the length-scale which is used to calculate time-steps (in Eq. (25)). We will define the mesh used in this paper in Sect. 3.1.

It is important to note that no special method is applied to ensure that Eq. (5) is satisfied throughout any MHD evolution. It follows that numerical errors naturally result in non-zero $\boldsymbol{\nabla} \cdot \boldsymbol{b}$ in more or less extended regions during the runs. However, these errors do not result in numerical instabilities, probably because the MHD equations are written in their fully developed form in our code, so that no non-physical explicit term due to $\boldsymbol{\nabla} \cdot \boldsymbol{b} \neq 0$ exists. The measurement of the divergence of magnetic field relative to the other magnetic field gradients provides a measurement of the error in the evaluation of gradients. The analysis of this error is reported in Sect. 4.7. 


\subsection{Explicit diffusion terms}

Using the true viscous stress tensor or more simply a classical Laplacian for the velocity diffusion would be a priori desireable. However, such terms are not appropriate for non-uniform meshes for the following two reasons. Firstly, they can overdiffuse at small scales where the cells are small, greatly reducing the advantage of using a non-uniform mesh. Secondly, they can under-diffuse at large scales where the cells are large, so that sharp gradients (e.g. shocks, shear layers) invariably lead to numerical instabilities.

So we chose rather to use only a pseudo-Laplacian diffusion term which is locally adapted to the mesh:

$\mathfrak{D} u_{i}=v^{\star}\left(\delta_{x}^{2} u_{i}+\delta_{y}^{2} u_{i}+\delta_{z}^{2} u_{i}\right)$

$v^{\star}=u_{v^{\star}} / d$,

where $u_{i}$ is the velocity component along either axis $(x ; y ; z), v^{\star}$ is a pseudo-viscosity and $u_{v^{\star}}$ is the characteristic speed (used as a free parameter in our code, see Sect. 3.4), and $\delta_{x}^{2}$ is a secondderivative operator with respect to the mesh rather than to spatial units. For any quantity $f$, this operator is equal to:

$\delta_{x}^{2} f=f\left(x^{i+1} ; y^{j} ; z^{k}\right)-2 f\left(x^{i} ; y^{j} ; z^{k}\right)+f\left(x^{i-1} ; y^{j} ; z^{k}\right)$.

This type of filter results in a Reynolds number equal to $u / u_{v^{\star}}$ on the scale of the mesh.

For numerical stability, the inclusion of a diffusive term for the magnetic field is necessary. However, the pseudo-Laplacian defined above cannot be used for arbitrary magnetic fields for the following reason. Consider for example a potential field which is by definition in equilibrium and which satisfies $\boldsymbol{\nabla}^{2} b_{i}=0$. This field does not satisfy $\mathfrak{D} b_{i}=0$, so that the use of $\mathfrak{D}$ will diffuse the field and generate artificial Lorentz forces. So our magnetic field diffusion term is the standard collisional (Laplacian) resistive term:

$\Re b_{i}=\eta\left(\partial_{x}^{2} b_{i}+\partial_{y}^{2} b_{i}+\partial_{z}^{2} b_{i}\right)$

$\eta=u_{\eta} d$

where $b_{i}$ is the magnetic field component along either axis $(x ; y ; z), \eta$ is the plasma resistivity and $u_{\eta}$ is a characteristic speed which is later used to set $\eta$. Setting $u_{\eta}=u$ would imply a magnetic Reynolds number equal to 1 at the smallest scale $d^{i, j, k}$. In the case of a non-uniform mesh, in principle this choice does not permit us to calculate configurations which would form fine current layers in the largest cells. However, in the range of applications studied in this paper (where the strongest magnetic field gradients developed in the smallest cells), and considering the non-linear coupling between $\boldsymbol{u}$ and $\boldsymbol{b}$, this choice is satisfactory.

In the applications studied in this paper, no additional explicit diffusive term for the density needed to be used.

\subsection{Spatial scheme}

Our code calculates third-order, five-point centered spatial derivations in non-uniform meshes. For each variable $f=\left(\rho ; u_{x} ; u_{y} ; u_{z} ; b_{x} ; b_{y} ; b_{z}\right)$, the expressions for the derivatives (e.g. along the $x$ axis) are directly calculated in the code as follows:

$\partial_{x} f^{i}=\frac{F^{i} I^{i}-E^{i} J^{i}}{F^{i} G^{i}-E^{i} H^{i}}$

$\partial_{x}^{2} f^{i}=2 \frac{G^{i} J^{i}-H^{i} I^{i}}{F^{i} G^{i}-E^{i} H^{i}}$

The coefficients result from linear combinations of four Taylor expansions, and are equal to:

$$
\begin{aligned}
& A^{i}=d x^{i}+d x^{i+1} \\
& B^{i}=d x^{i} \\
& C^{i}=d x^{i-1} \\
& D^{i}=d x^{i-1}+d x^{i-2} \\
& E^{i}=A^{i 2} B^{i 3}-A^{i 3} B^{i 2} \\
& F^{i}=C^{i 3} D^{i 2}-C^{i 2} D^{i 3} \\
& G^{i}=A B^{i 3}-A^{i 3} B \\
& H^{i}=C D^{i 3}-C^{i 3} D \\
& I^{i}=B^{i 3}\left(f^{i+2}-f^{i}\right)-A^{i 3}\left(f^{i+1}-f^{i}\right) \\
& J^{i}=C^{i 3}\left(f^{i-2}-f^{i}\right)-D^{i 3}\left(f^{i-1}-f^{i}\right) .
\end{aligned}
$$

These coefficients are functions of the intervals between gridpoints (given in Eq. (13)), and of the values of $f$ at the point where the derivative is calculated and at its four closest neighbours in the $x$ direction.

Such a high order scheme gives precise derivatives, but is not appropriate for dealing with sharp discontinuities on the scale of the mesh. Therefore we have to adjust the diffusion coefficients $\left(u_{v^{\star}} ; u_{\eta}\right)$ defined in Sect. 2.2 so as to ensure that every gradient is resolved over at least four grid points.

Equations (24) show that some values "outside of the domain" need to be specified for calculating derivatives at the boundary and at the first point above it. This is achieved by the inclusion of two layers of ghost cells at each boundary, whose distances to the boundary are equal to their mirrors inside the domain, and whose values are specified accordingly with the type of boundary (see Sects. 2.5 and 2.6). The total number of gridpoints which needs to be specified is thus $\left(n_{x}+4 ; n_{y}+4 ; n_{z}+4\right)$.

\subsection{Time scheme}

Our time integration scheme is of the so-called "predictorcorrector" family. It is calculated by linear combinations of Taylor expansions of time derivatives at several time steps. At each step $n$ corresponding to a physical time $t^{n}$, the calculation of the right-hand side of Eqs. (6)-(12) for every quantity $f=\left(\rho ; u_{x} ; u_{y} ; u_{z} ; b_{x} ; b_{y} ; b_{z}\right)$ first results in their time derivatives: $\partial_{t} f^{n}$.

Then the timestep $\mathrm{d} t^{n}$ to reach the time $t^{n+1}=$ $t^{n}+\mathrm{d} t^{n}$ is dynamically adjusted according to the standard Courant-Friedrich-Levy condition:

$\mathrm{d} t^{n}=C_{\mathrm{CFL}} \min \left(\frac{d^{i, j, k}}{u+b / \sqrt{\mu \rho}}\right)$, 
where $C_{\mathrm{CFL}}<1$ ensures that during $\mathrm{d} t^{n}$ no information propagates along distances larger than any cell. For every calculation in this paper, we use $C_{\mathrm{CFL}}=0.5$. Then the following variables are updated:

$A^{n}=\mathrm{d} t^{n}$

$B^{n}=\mathrm{d} t^{n-1}$

$C^{n}=\mathrm{d} t^{n-1}+\mathrm{d} t^{n-2}$

$D^{n}=\left(B^{n 2} C-B C^{n 2}\right)^{-1}$

$E^{n}=\left(A^{n 2} B+A B^{n 2}\right)^{-1}$.

Using these, a first-step explicit time integration is achieved, resulting in "predicted" values $f_{\mathrm{P}}^{n+1}$ for $f$ at the time $t^{n+1}$ :

$$
\begin{aligned}
f_{\mathrm{P}}^{n+1}= & f^{n}+A^{n} \partial_{t} f^{n}+\frac{1}{3} A^{n 3} D^{n}\left[C^{n} \partial_{t} f^{n-1}\right. \\
& \left.-B^{n} \partial_{t} f^{n-2}+\left(B^{n}-C^{n}\right) \partial_{t} f^{n}\right] \\
& +\frac{1}{2} A^{n 2} D^{n}\left[C^{n 2} \partial_{t} f^{n-1}-B^{n 2} \partial_{t} f^{n-2}\right. \\
& \left.+\left(B^{n 2}-C^{n 2}\right) \partial_{t} f^{n}\right] .
\end{aligned}
$$

This integration requires the use of the timesteps and the time derivatives of $f$ at three times before the time which is to be reached. It is called a 3-step Adams-Bashforth method, allowing time-varying timesteps.

Using $f_{\mathrm{P}}^{n+1}$, Eqs. (6)-(12) are calculated again, resulting in $\partial_{t} f_{\mathrm{P}}^{n+1}$. These are now used in a second step, where the values $f_{\mathrm{P}}^{n+1}$ are "corrected" to their final value $f^{n+1}$ :

$$
\begin{aligned}
f^{n+1}= & f^{n}+A^{n} \partial_{t} f^{n}+\frac{1}{3} A^{n 3} E^{n}\left[B^{n} \partial_{t} f_{\mathrm{P}}^{n+1}\right. \\
& \left.+A^{n} \partial_{t} f^{n-1}-\left(A^{n}+B^{n}\right) \partial_{t} f^{n}\right] \\
& +\frac{1}{2} A^{n 2} E^{n}\left[B^{n 2} \partial_{t} f_{\mathrm{P}}^{n+1}-A^{n 2} \partial_{t} f^{n-1}\right. \\
& \left.+\left(A^{n 2}-B^{n 2}\right) \partial_{t} f^{n}\right] .
\end{aligned}
$$

This integration requires the use of the timesteps and the time derivatives at the initial time and one step before, as well as at the time which is to be reached. It is called a 2-step Adams-Moulton method.

This method yields more precise and stable results than a simple Adams-Bashforth method, although it is timeconsuming because the MHD equations have to be solved twice for each given time-step.

\subsection{Open boundary conditions}

In the solar application studied in this paper, some of our boundaries need to be transparent, so as to allow information to go out of the numerical domain, since the corona is a physically open system, apart from its bottom. Using standard hardwall with or without free-slip boundaries would naturally lead to wave and bulk-flow reflexions from these boundaries back into the domain, which would lead in particular to an artificial confinement of the system.

Open boundary conditions are often treated as follows when the characteristics are not used. Each variable is simply copied from the boundary value into the external ghost cells. However, Eqs. (7)-(9) show that this method is not sufficient in general. Consider a magnetic field which is in equilibrium analytically, but whose gradients are not zero near the boundaries (e.g. as it is the case for a dipole field). The ghost cells defined as above will inevitably result in spurious Lorentz forces which will accelerate the plasma from the boundaries. We tested this and found that, for the initial setting described in Sect. 3, the plasma quickly reached inflowing (followed by outflowing) velocities of the order of the Alfvén speed. These velocities then propagated into the domain and thus strongly influenced the system. In order to get rid of this effect, we only integrate Eqs. (7)-(9) where all the quantities required to calculate derivatives are specified within the domain, and not in the ghost cells.

In our code, "open" boundaries are achieved by copying all variables $\left(\rho ; b_{x} ; b_{y} ; b_{z}\right)$ from their value at the boundary onto the two external ghost cells, while the values of $\left(u_{x} ; u_{y} ; u_{z}\right)$ at two gridpoints within the domain away from the boundary are copied not only onto the ghost cells, but also onto the boundary itself and onto the first point within the domain. This results in an artifical decrease of gradients close and perpendicularly to the boundary.

This method allows a structure to leave the box without numerical instability as long as the physical width of this structure is larger than several grid points. However, it leads to some noise on the scale of the mesh near the boundary, whose amplitude depends on the length-scale of the exiting structure. It must be noted that this method also allows inflows, which when they occur bring into the domain zero-gradient quantities, as specified in the ghost cells. In our present applications, all the noise which developed at the open boundaries remained - at most - ten times lower than the signal, and was efficiently damped by the diffusion terms. Also, the existing inflows did not seem to play a significant role in the evolution of the system.

\subsection{Line-tied boundary conditions}

We use so-called "line-tied reflective boundary conditons" which ensure that the footpoint of a magnetic field line can only move horizontally onto the boundary, and only if this motion is prescribed kinematically. Physically, this corresponds to an infinitely conducting and inertial plane, which cannot be forced by what happens in the domain. Such a boundary can be used to simulate the interface between an accretion disk or the interior of a star, with its surrounding diffuse corona. In the frame of solar applications it is taken to be the photosphere (see Sect. 1).

In a general case for which the magnetic field is neither purely tangential nor purely normal to the boundary, the standard Dirichlet-Neumann boundary (or parity) conditions cannot be used for every variable at a line-tied plane. 
In our code, this boundary is placed in the plane $\left(x^{i} ; y^{j} ; z^{k=1}\right)$, and is specified as follows. At $k=1$, Eqs. (7)(9) are not solved and the velocities are given by:

$u_{z}^{1}=0$

$u_{x}^{1} ; u_{y}^{1}=0$ or prescribed

$u_{v^{\star}}^{1} ; u_{\eta}^{1}=0$.

Note that the explicit diffusion terms are set to zero in this plane. The values of $\left(\rho^{k} ; u_{z}^{k}\right)$ in the two ghost cells $(k=1-m$ and $m=1 ; 2)$ are imposed so as to ensure symmetry for $\rho$ and antisymmetry for $u_{z}$ :

$\rho^{1-m}=\rho^{1+m}$

$u_{z}^{1-m}=-u_{z}^{1+m}$.

The ghost cells are specified for the remaining variables $f=$ $\left(b_{x}^{k} ; b_{y}^{k} ; b_{z}^{k} ; u_{x}^{k} ; u_{y}^{k}\right)$ so that:

$f^{1-m}=2 f^{1}-f^{1+m}$.

These result in antisymmetric conditions for the transverse components of the velocities when they are set to zero.

In spite of their complexity and of the sharp transition which exists between the boundary and the domain (in which Eqs. (7)-(9) are solved, with non-zero diffusion terms), these settings provide a numerically stable boundary which satisfies the line-tied and reflective conditions with no directly noticeable boundary layer effect, as long as the scale-length of any variable is larger than a few gridpoints. However, note that these settings result in the formation of non-zero $\boldsymbol{\nabla} \cdot \boldsymbol{b}$ near the boundary (see Sect. 4.7).

\section{Numerical settings}

In this section, we describe the initial parameters which are chosen to perform our calculations, and the procedures which were applied to perform both continuously driven and relaxation runs.

\subsection{Mesh and initial magnetic field and density}

We construct our initial conditions in two steps. First we define an analytical vertical magnetic field $b_{z}$ at the line-tied (photospheric) boundary $z=0$ as two circular gaussians of opposite flux:

$$
\begin{aligned}
b_{z}(x ; y ; z=0)= & b_{0} \exp \left(-\frac{\left(x-x_{0}\right)^{2}+y^{2}}{r_{0}^{2}}\right) \\
& -b_{0} \exp \left(-\frac{\left(x+x_{0}\right)^{2}+y^{2}}{r_{0}^{2}}\right) .
\end{aligned}
$$

Even though our code uses dimensionalized quantities in MKSA, in the following the magnetic field will always be expressed in $\mathrm{G}$ and the distances in $\mathrm{Mm}$, which are convenient units for solar aplication. We set $b_{0}=650 \mathrm{G}, x_{0}=8 \mathrm{Mm}$ and $r_{0}=15 \mathrm{Mm}$.

This synthetic magnetogram is used to set boundary conditions for a potential field extrapolation $(\boldsymbol{\nabla} \times \boldsymbol{b}=\mathbf{0})$ using

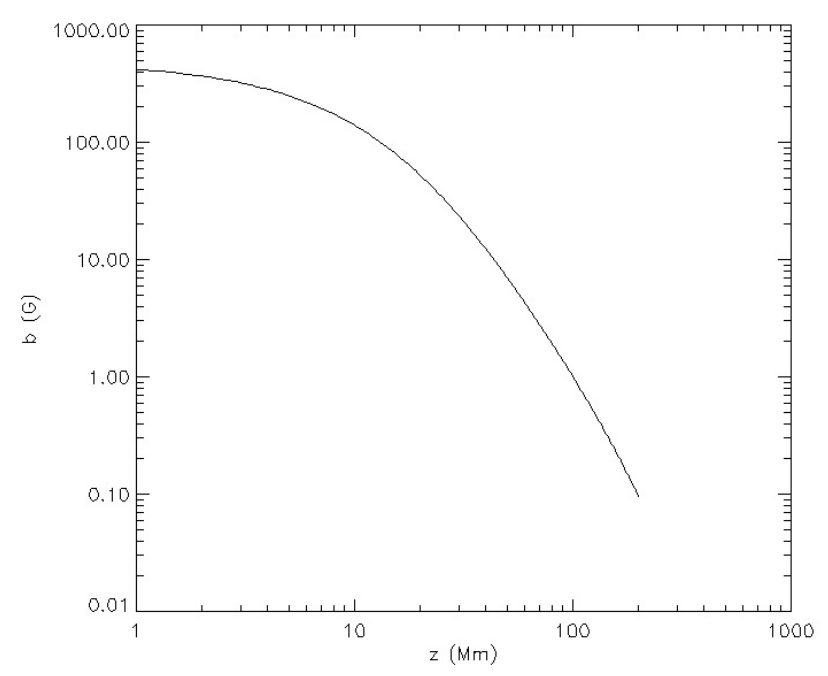

Fig. 1. Magnitude of the initial potential magnetic field versus altitude at the center of the domain at $(x ; y)=(0 ; 0)$.

the Fourier code developed by Démoulin et al. (1997) which is periodic in $(x ; y)$. The extrapolation is done with $N_{x} \times N_{y}=$ $1024^{2}$ points uniformly distributed on the $(x ; y)$ plane with $(x ; y) \in[-200 \mathrm{Mm} ; 200 \mathrm{Mm}]$. The extrapolation results in a magnetic field decrease with $z$ which is plotted in Fig. 1.

In order to calculate an open rather than a periodic system in our MHD runs, a domain $\mathcal{D}$ is extracted from the periodic domain used in the extrapolation. In the following, we consider $\mathcal{D}$ in $(x ; y) \in[-100 \mathrm{Mm} ; 100 \mathrm{Mm}]$ and $(z) \in$ [0 Mm; $200 \mathrm{Mm}]$. Using linear interpolations between the gridpoints of the Fourier extrapolation, the magnetic field values are saved on a non-uniform mesh with $n_{x} \times n_{y} \times n_{z}=$ $201^{3}$ points. The smallest cells are concentrated around $x=$ $y=z=0$, on top of the inversion line of $b_{z}(z=0)$ where $\left(b_{x} ; b_{y}\right)$ are the strongest at $t=0$. The mesh intervals vary in the range $(\mathrm{d} x ; \mathrm{d} y ; \mathrm{d} z) \in[0.2 \mathrm{Mm} ; 2.8 \mathrm{Mm}]$, expanding from $x=y=z=0$ following $d_{x}^{i+1} / d_{x}^{i}=d_{y}^{j+1} / d_{y}^{j}=1.027$ and $d_{z}^{k+1} / d_{z}^{k}=1.013$. The resulting potential field is shown in Fig. 2.

As a result of the extrapolation, the ratio of the maximal to the minimal value of $b$ in $\mathcal{D}$ is about $5 \times 10^{3}$. In order to mimic (1) the filling of plasma in coronal loops rooted in strong field regions, (2) the coronal density decrease with altitude and (3) the fact that the Alfvén speeds $c_{\mathrm{A}}$ always remain much larger in the corona than the photospheric driving velocities, we prescribe the ad-hoc initial density in $\mathcal{D}$ :

$\rho(x ; y ; z ; t=0)=\left(\mu c_{\circ}\right)^{-1} b^{2}(x ; y ; z ; t=0)$,

so that $c_{\mathrm{A}}(x ; y ; z ; t=0)=c_{0}=10^{3} \mathrm{~km} \mathrm{~s}^{-1}$. In all our runs, we find that local magnetic field and density variations result in Alfvén speed variations roughly between 0.7 and $5 c_{0}$. These settings and Eq. (25) result in timestep values of $0.01<\mathrm{d} t(\mathrm{~s}) \leq$ 0.1 .

\subsection{Procedure for boundary driving}

We apply a horizontal divergence-free boundary driving of the field line footpoints at $z=0$ so as to kinematically twist the 

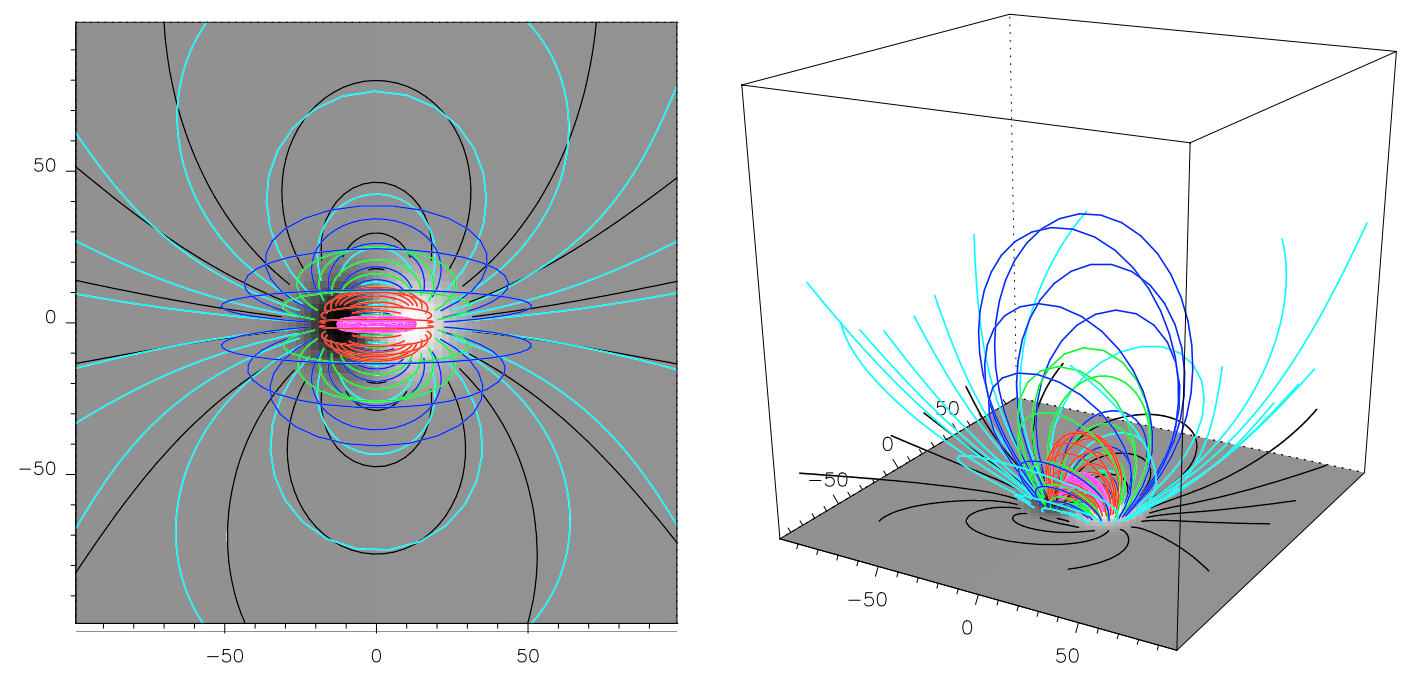

Fig. 2. Top and projection views of the initial potential magnetic field within the whole domain. The grey scale at the $z=0$ plane is white/black for $b_{z}= \pm 480 \mathrm{G}$. [Pink; red; green; dark-blue; light-blue; black] field lines are rooted in $b_{z}= \pm[475 ; 400 ; 300 ; 250 ; 150 ; 50] \mathrm{G}$.
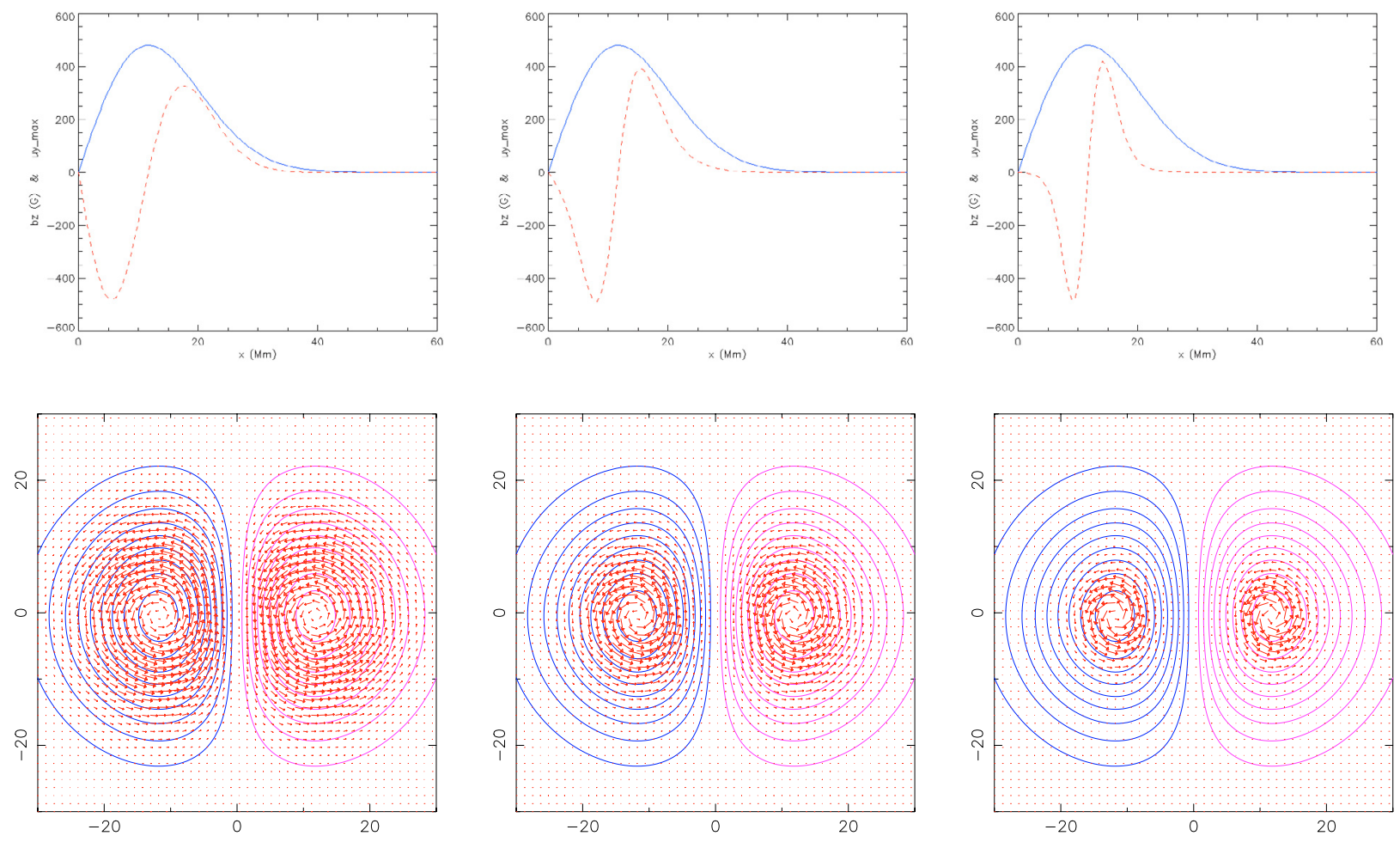

Fig. 3. (First row): Profiles of $b_{z}$ and $u_{y}$ along the $(x ; y=0, z=0)$ axis. (Second row): Contours of $b_{z}= \pm 50 ; 100 ; 150 ; 200 ; 250 ; 300 ; 350$; 400; $450 \mathrm{G}$ and velocity vectors at $z=0$. The [first;second; third] columns correspond to vortices defined by $\zeta=[10 ; 0.9 ; 0.5]$.

initial potential configuration. We choose a velocity field which preserves the initial distribution of $b_{z}(z=0)$, so that the minimum magnetic energy remains the same during all runs, i.e. the energy of the potential field at $t=0$. In this context, Eq. (12) at $z=0$ turns into:

$\partial_{t} b_{z}=-\nabla_{\perp} \cdot\left(b_{z} \boldsymbol{u}_{\perp}\right)$

where $\boldsymbol{u}_{\perp}=\left(u_{x} ; u_{y}\right)$ and $\boldsymbol{\nabla}_{\perp}=\left(\partial_{x} ; \partial_{y}\right)$. Eq. (37) then implies:

$\boldsymbol{u}_{\perp}=\nabla_{\perp} \psi\left(b_{z} ; t\right) \times \boldsymbol{e}_{z}$ where $\boldsymbol{e}_{z}$ is the unit vector along the $z$ axis and $\psi\left(b_{z} ; t\right)$ is an arbitrary potential which only depends on $b_{z}$ and on time. In all our runs we choose the same potential as Amari et al. (1996) and Török \& Kliem (2003):

$\psi\left(b_{z} ; t\right)=\psi_{\circ} \gamma(t) b_{z}^{2} \exp \left(\frac{b_{z}^{2}-b_{z}^{\max 2}}{\zeta^{2} b_{z}^{\max 2}}\right)$,

where $\gamma(t)$ is a ramp function giving an initial acceleration toward a constant twisting velocity:

$\gamma(t)=\frac{1}{2} \tanh \left[\frac{2\left(t-t_{\alpha}\right)}{t_{\omega}}\right]+\frac{1}{2}$ 
This function results in two vortices centered around the maxima of $\left|b_{z}(z=0)\right|$ for which the horizontal velocity vectors are tangential to the contours of $b_{z}(z=0)$. In our runs, we fix $\psi$ 。 so that $\boldsymbol{u}_{\perp}^{\max }=20 \mathrm{~km} \mathrm{~s}^{-1}=0.02 c_{0}$. The middle of the ramp function is at $t_{\alpha}=300 \mathrm{~s}$ and its half-width is $t_{\omega}=100 \mathrm{~s}$. This ramp function allows the system to find a good numerical equilibrium before $t=200 \mathrm{~s}$, when the acceleration begins. $\zeta$ is a free parameter which controls the physical extension of the vortices. Even though this boundary driving analytically preserves $b_{z}(z=0)$, it numerically results in small deformations of $b_{z}(z=0)$ contours in the vicinity of the vortex centers. In order to ensure that $b_{z}(z=0)$ is conserved in our runs, it is numerically re-enforced at each time-step.

In order to study the dependence of the system on the size of vortices, we performed three sets of runs, with $\zeta=10$; $0.9 ; 0.5$. The first case results in extended vortices which twist most of the flux and which shear the inversion line, the third case results in concentrated vortices which twist only the strongest fields, and the second case is intermediate. The associated velocity fields are shown in Fig. 3.

\subsection{Procedure for relaxation runs}

Even though the applied boundary driving is very slow (2\% of the Alfvén speed), the continuously twisted runs always end up after some time in velocities of the order of $c_{\mathrm{A}}$ (see Sect. 4). This indicates that the system is far from equilibrium.

In order to find an equilibrium (or to reveal its absence) for a given amount of twist reached at a time $t^{\star}$, we perform relaxations runs as follows. Time is reset to zero, the magnetic field and mass density are set to $[\boldsymbol{b} ; \rho](t=0)=[\boldsymbol{b} ; \rho]\left(t=t^{\star}\right)$, the boundary driving is suppressed $\left(\psi_{\circ}=0\right)$ and the velocities are reset to $\boldsymbol{u}(t=0)=\mathbf{0}$. So the further evolution of the system is only driven by the residual Lorentz forces which exist at $t=$ $t^{\star}$ during the continuously driven run.

This method is more demanding numerically than applying a gradual deceleration of the driving at $z=0$ while keeping its velocities in $\mathcal{D}$. However, it is more convenient because the amount of twist is fixed during the relaxation (if $u_{\eta}$ is small enough), and essentially because velocities resulting from the accumulation of momentum during the driven phase do not play a role in the evolution of the system. This issue becomes important when the velocities are of the order of the Alfvén speed.

\subsection{Diffusion parameters}

In order to avoid the formation of unresolved strong shear layers at high altitudes $z$, which naturally appear due to prescribed motions at the footpoints of strongly expanding flux tubes, we apply a strong diffusion term for $\boldsymbol{u}: u_{v^{\star}}=150 \mathrm{~km} \mathrm{~s}^{-1}$. However, to ensure a high magnetic Reynolds number in the corona, we set a much smaller diffusion term for $\boldsymbol{b}: u_{\eta}=15 \mathrm{~km} \mathrm{~s}^{-1}$.

Estimating $(i)$ the typical length-scale as $30 \mathrm{Mm}$, which is roughly equal to the initial length of the magnetic field line rooted in $b_{z}^{\max }(x ; y ; z=0)$ as well as the average transverse extension of the twisted flux tube which forms in our runs; and (ii) typical velocities as $0.1 c_{0}$, the characteristic dimensionless numbers are $\left(R_{e} ; R_{m} ; L_{u}\right)=\left(10^{2} ; 10^{3} ; 10^{4}\right)$. The value of the Reynolds number here is only an upper limit, since the viscous term is defined at the scale of the mesh in our code (see Eq. (17)).

The diffusion velocities $\left(u_{\eta} ; u_{v^{\star}}\right)$ are kept constant in space and time, and are the same for every run (continuously driven, relaxation and varying $\zeta$ ).

\section{Results from the continuously twisted runs}

\subsection{Twist definition and maximum values}

During the continuous photospheric driving, field lines rooted in the prescribed vortices start to twist around the "axial field line" which is rooted in $b_{z}^{\max }$ at the vortex centers. Figure 4 shows that both the magnetic and kinetic energies increase monotonically for the three vortex sizes considered $(\zeta=$ $10 ; 0.9 ; 0.5)$ as long as the boundary driving is maintained. The kinetic energy for the small vortices naturally remains smaller than the one for large vortices, since the coronal volume at $z>0$ which is affected by the vortices depends on the vortex size.

Since the three vortices have different sizes, but with the same maximum velocity and initial acceleration ramp, they have different rotation rates around the axial field line. So in the following, we study the variation of all quantities as a function of twist $\Phi$ rather than time, so as to have a common timescale. $\Phi$ is calculated analytically, and also checked numerically at various timesteps by integrating many magnetic field lines along the $x$ axis for $(x<0 ; y=0 ; z=0)$ and by measuring the rotation rate of their footpoints at $(x>0 ; z=0)$. We find that the prescribed motions result in a nearly rigid rotation inside a small radius $R_{\text {phot }} \simeq 1.5 \mathrm{Mm}$ around the vortex centers, which gradually decreases away from them. The twist unit $\Phi$ which we consider in the rest of the paper is the rotation angle of the field line footpoints in the small area which is subject to a quasi-rigid rotation. We define the number of turns in the associated magnetic flux tube by $\mathcal{N}=\Phi / 2 \pi$.

For the three vortex sizes considered, the results of the calculations are only shown up to twists equal to $\mathcal{N}^{\max }=$ $(1.22 ; 1.61 ; 1.66)$ turns for $\zeta=(10 ; 0.9 ; 0.5)$ for the following reasons. The physical meaning of the runs is indeed bounded by two limitations. Firstly, the most relevant field lines (those anchored in strong field regions) can reach (and then cross) the top boundary of the domain, which prevents communication via Alfvén waves between their footpoints at $z=0$. This limitation comes up for $\zeta=10$ when $\mathcal{N}>\mathcal{N}^{\max }$, during continuously driven runs, and for $\zeta=0.9$ when $\mathcal{N}>1.48$ during relaxation runs (see next subsections). Secondly, a current shell develops in all runs for $z \geq 0$ at the interface between the strongly and weakly twisted field lines : this shell must remain numerically resolved. For $\zeta=0.5$ the shell becomes unresolved for $\mathcal{N}>1.70$, simply because the vortices are more concentrated than in the other runs. This leads to numerical instabilities which start to develop around $(x ; y ; z)=( \pm 9 ; \pm 17 ; 30) \mathrm{Mm}$, where the cell intervals (defined by Eq. (13)) are $(\mathrm{d} x ; \mathrm{d} y ; \mathrm{d} z) \sim$ $(0.3 ; 0.6 ; 0.6) \mathrm{Mm}$. Increasing the resistivity makes it possible 

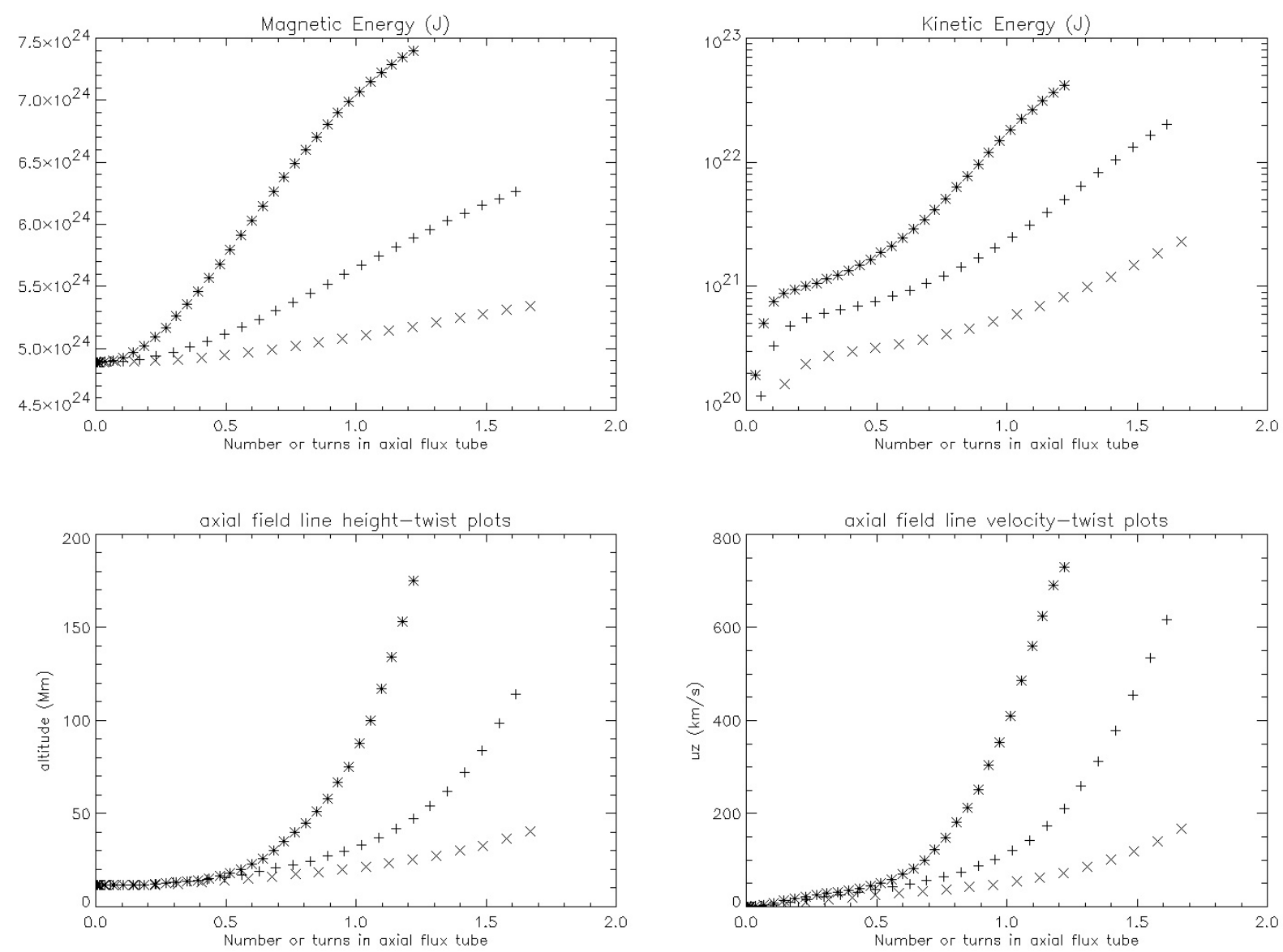

Fig. 4. Continuously twisted runs. All quantities are plotted as a function of the central twist $\mathcal{N}$. Each mark is separated in time by $\Delta t=30 \mathrm{~s}$. [stars; pluses; crosses] correspond to $\zeta=[10 ; 0.9 ; 0.5]$. (First row): Global magnetic and kinetic energies. (Second row): Altitude and vertical velocity of the axial field line rooted in the vortex centers.

to bypass this problem. However, we did not go on in this direction, because comparison with other runs would have been difficult.

\subsection{Quasi-static phase}

During a first long quasi-static period, in the strongest and closed field regions, the slow velocities of the photospheric driving allow the low frequency Alfvén waves which are generated at $z=0$ by the vortices to travel several times along a given field line by rebouncing on the line-tied plane at $z=0$. This leads to a smooth distribution of the generated electric currents along each magnetic field line. This allows the magnetic configuration to remain close to a non-linear force-free state. This was checked by integrating both electric current and magnetic field lines, which become undistinguishable from one another after a short transition phase after the start of the runs. In the outer "open" field lines rooted in weak field regions (which emerge out of the numerical domain) the waves are transmitted through the open boundaries with very little reflexion, thanks to the dissipative operator for the velocity applied on the non-uniform mesh. However, since these field lines are also continuously twisted, they do not come back to a potential state.
The whole magnetic configuration slowly expands in all directions, but mostly along $z$. This can be explained in the context of the van Tend \& Kuperus (1978) model by the generation of surface currents at the infinitely conducting and inertial plane at $z=0$, which tend to expell the generated coronal currents away from them, and by the self-repulsion of the coronal currents for $z>0$. This can also be explained by the fact that since $b_{z}(z=0)$ remains constant, the currents at $z>0$ generated by the twisting motions at $z=0$ result in a global increase of magnetic energy, hence in local enhancement of magnetic pressure within the twisted flux tube, so in local expansion where the magnetic tension does not increase as fast as the magnetic pressure. During this quasi-static phase, the twisting flux tube expands with a vertical velocity $u_{z}$ which is smaller than the boundary driving maximal velocity. This first phase lasts until the accumulated vertical velocities become of the order of $5 \%$ of the Alfvén speed, i.e. $u_{z}=50 \mathrm{~km} \mathrm{~s}^{-1}$, which is nearly equal to the sum of the two vortex velocities, which is about the maximum amplitude of the torsional Alfvén wave propagating along the loop.

\subsection{Dynamic phase}

A second phase of dynamic nature then begins. The rates of increase for $u_{z}$, for the kinetic energy and for the apex altitude of 
the axial field line gradually rise to much stronger values than during the quasi-static phase. This smooth transition does not occur for the same twist for all vortex sizes. Figure 4 shows that the change occurs for central twists of $\mathcal{N}^{\star} \sim 0.5 ; 0.7 ; 1.0$ turns respectively for $\zeta=10 ; 0.9 ; 0.5$. As the twist increases, the kinetic energy gains one order of magnitude, and the vertical velocity of the axial field line reaches a non-negligible fraction of the Alfvén speed. For a given twist the vertical velocity of large overlaying field lines is even higher (but always sub-Alfvénic in our runs): these large field lines are pushed upward and sideward together, both due to their own internal currents and to the pushing from below by the more twisted flux tube. Analyzis of the height-time curves for the axial field line reveals an expansion rate which is slightly faster than exponential. During this second phase the magnetic field configuration becomes less and less force-free, as indicated not only by the very fast expansion velocities, but also by an increasing angle between the electric current and the magnetic field lines.

Regardless of the existence or not of a non-equilibrium, the observed departure from the force-free state is expected. Indeed, the vertical expansion results in longer and longer field lines. The rate of increase of field line length becomes such that the low frequency Alfvén waves emitted from the photospheric boundary at $z=0$ no longer have time to rebounce several times along a given field line, so that the currents cannot be quasi-statically distributed all along the field line. This results in a system which is always trying to reach an equilibrium (if any) that is located at larger and larger heights in $z$ as the twist increases.

As the twist increases, the core twisted flux tube emerges out the numerical domain at a rate which is faster than exponential, with velocities of the order of the Alfvén speed. Meanwhile, the surrounding twisted and potential loops lean sidewards. This whole behaviour described above is qualitatively the same for all three vortex sizes considered in this paper. It is also fully consistent with what was reported for other classes of bipolar twisted fields calculated by Amari et al. (1996) and Török \& Kliem (2003) with different zero- $\beta$ MHD codes. Consequently, we conclude that the "very fast opening" initially reported by Amari et al. (1996) is a generic property of continuously twisted bipolar line-tied flux tubes.

\subsection{Influence of the weak field regions}

The comparison of the height-time plots for the three vortex cases during both quasi-static and dynamic phases shows that the vertical expansion rate of the axial field line is much larger for large than for small vortices. The dependence on vortex size is much larger than linear. Since the outer field lines are twisted more by the large vortices, we interpret the above result as indicating that the degree of twist in the outer field lines has a large influence on the expansion rate of the strong fields.

This can be qualitatively explained by the expansion of the outer field lines under the action of their own internal currents, which adds up to pushing from below due the twisted flux tube expansion. Quantitatively, the high sensitivity to the vortex size may be associated with the behaviour of large field lines in constant- $\alpha$ linear force-free field models, whose distortion and expansion rates are typically much larger than those of lowlying field lines for small increments of $\alpha$, as explained below.

In $(x ; y)$ periodic linear force-free field models, the magnetic field amplitude varies with $z$ as $b(z) \propto \exp \left(-z \sqrt{k^{2}-\alpha^{2}}\right)$ where $k=2 \pi / L$ is the wavenumber of the considered Fourier mode (see e.g. Aulanier \& Démoulin 1998). This readily shows that low-wavenumber modes (i.e. large field lines) are much more affected by $\alpha$ variations (i.e. by injection of electric currents) than high-wavenumber modes.

Even though this explanation cannot be directly transposed to the present MHD calculations since they result in nonconstant $\alpha$ distributions and in departures from the force-free state, we believe that it is at the origin of the strong dependence of the system on the twisting (or not) of large outer field lines.

\subsection{Inclination and reorientation of the field lines}

Figure 5 shows the structure of the magnetic configuration for the three vortices at the same twist during the dynamic phase. These figures show the sideward inclination of the field lines which surround the twisted core (drawn with pink field lines) and which are rooted in weak field regions on the edge of the photospheric bipole. This behaviour is similar to what Amari et al. (1996) reported.

A re-orientation of the core twisted flux tube is also evident, as found in the calculations of Török \& Kliem (2003). We find that this flux tube makes an angle with its initial orientation along the $x$ axis, which scales like the vortex size. However, this re-orientation is neither associated to the dynamic phase nor to some kink-like instability, since it is already noticeable at $\mathcal{N} \sim 0.5 \mathcal{N}^{\star}$ during the quasi-static phase. This phenomenon is in fact simply caused by the swirling of initially low-lying field lines having strong transverse fields which cross the inversion line. This swirling results in the increase of magnetic pressure along the $y$ axis at low altitude on one side of a given vortex. This pressure term is not present on the other side of the vortex since the corresponding larger field lines are relatively less deformed by the vortex. The pressure imbalance pushes the lower parts of the flux tube sideward from the $x$ axis, and this deformation propagates to larger heights along each field line thanks to Alfvén waves, which results finally in the deformation of the whole twisted flux tube.

\subsection{Behaviour near the top boundary}

When the core twisted flux tube, which is associated with the strongest volumic currents, gets close to the top boundary, it keeps accelerating even though the acceleration diminishes (see Fig. 4 for the run with $\zeta=10$ ). The apex of flux tube eventually always passes through the top boundary, just like some surrounding field lines rooted in weak field regions do at smaller times. The decrease of acceleration when the axial field lines passes $z \sim 175 \mathrm{Mm}$ is probably not physical, this altitude being only at 9 mesh points from the boundary. 

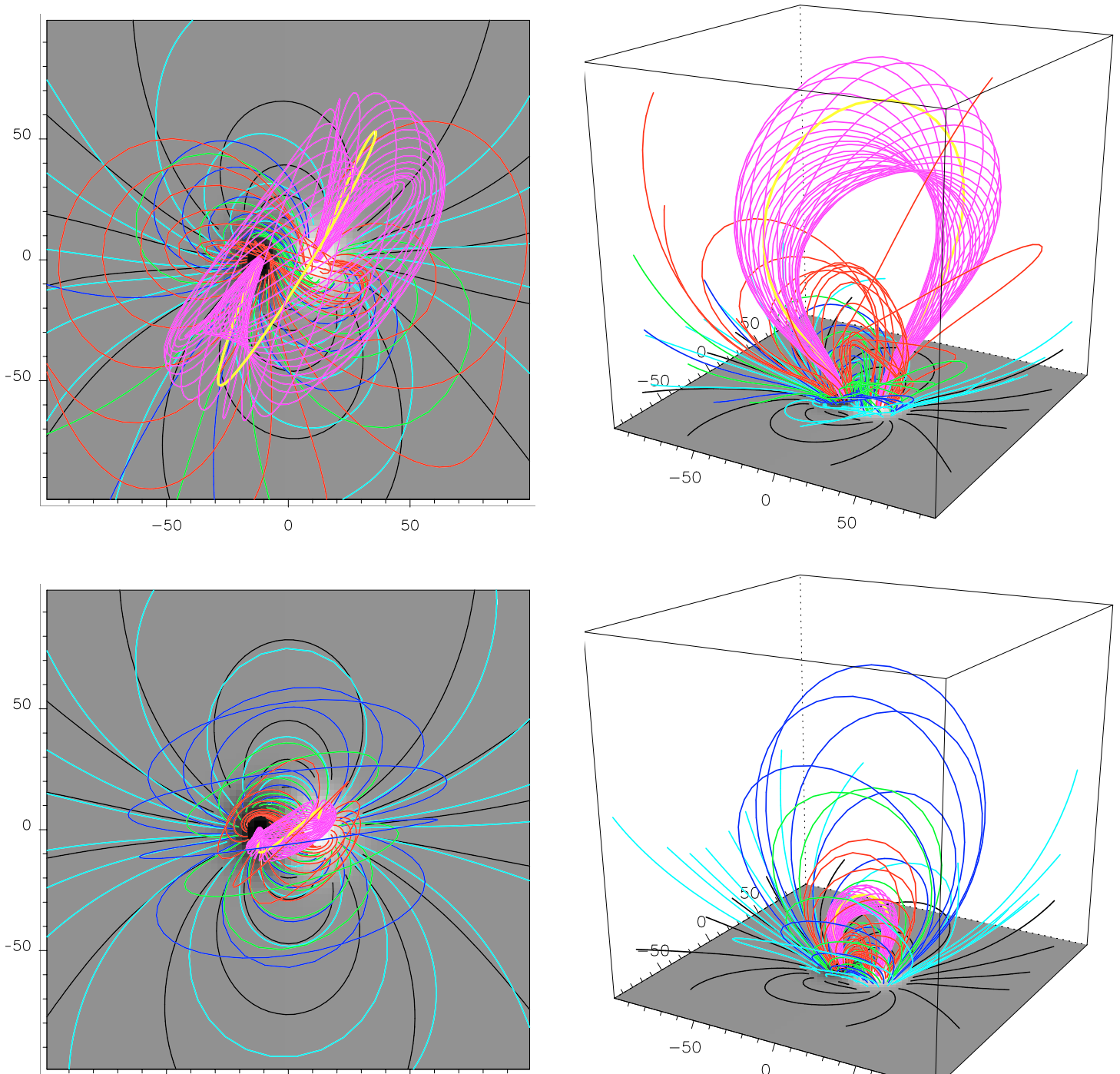

$-50$

0

50
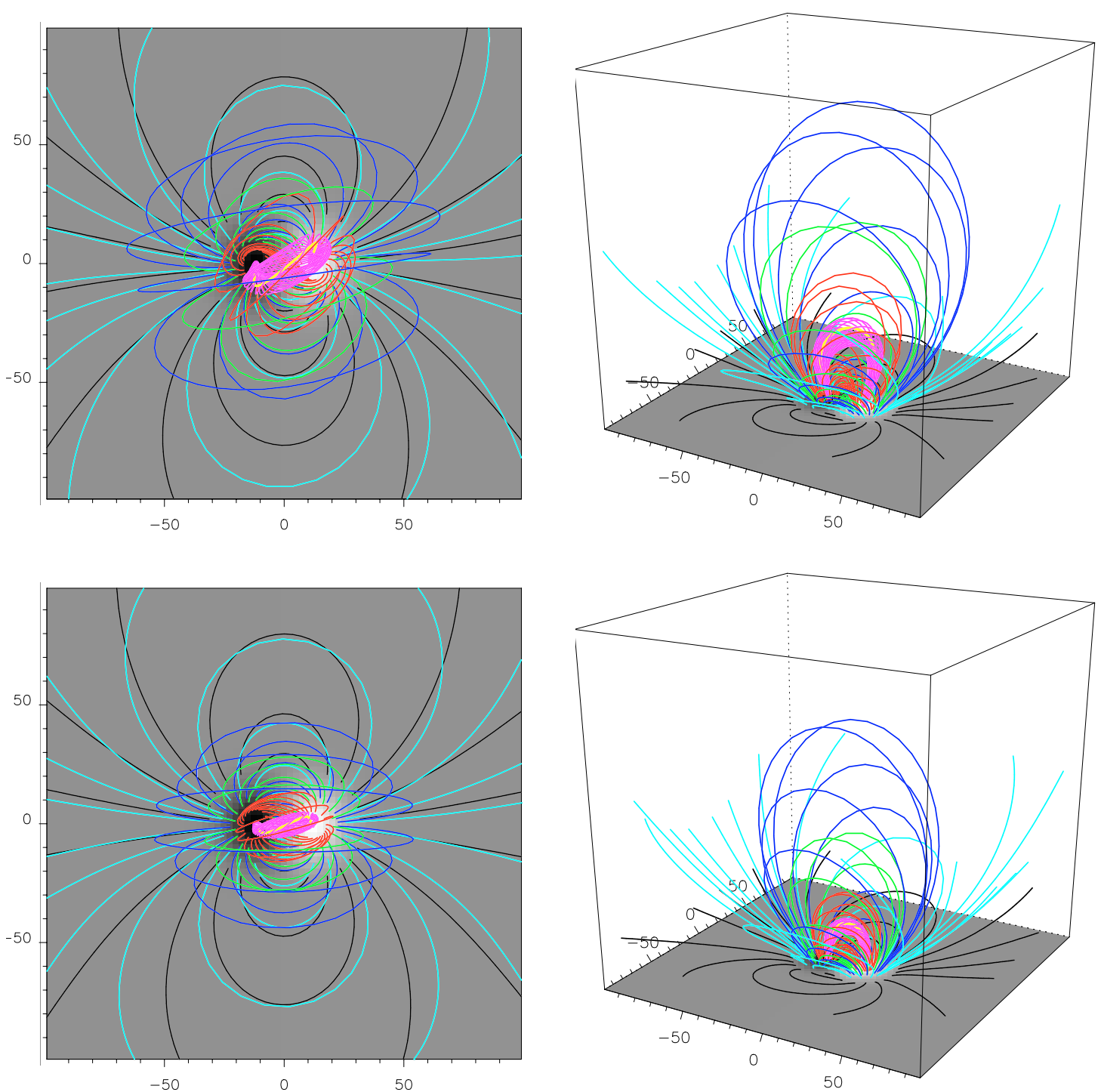

Fig. 5. Magnetic configurations for $\zeta=[10 ; 0.9 ; 0.5]$ shown on the [first; second; third $]$ row. All figures are plotted for a central twist $\mathcal{N} \sim$ 1.25 turns. The color coding is the same as in Fig. 2. 
It is plausible that this effect is a direct consequence of the condition which implies a zero-gradient condition for $\boldsymbol{u}$ at the open boundary (see Sect. 2.5). However, since all waves are strongly damped near the boundary because of the larger mesh size, this artificial reflexion should not significantly influence the system at lower heights, where the magnetic field is larger.

After the twisted flux tube exit, the vertical velocities in the domain start to fall, but always remain positive, thanks both to inertia and to the continuous photospheric driving which keeps injecting currents both in "open" field lines and in lower sheared/twisted arcades.

\subsection{Analyzis of the error in $\nabla \cdot b$}

As noted in Sects. 2.1 and 2.6, the solenoidal condition for the magnetic field is not fully ensured in our calculations. Even though this error is not noticeable in direct plots of various quantities, it can be estimated by calculating the ratio of $\boldsymbol{\nabla} \cdot \boldsymbol{b}$ to the local derivatives of the magnetic field:

$\mathcal{E}=\frac{\Sigma_{i} \partial_{i} b_{i}}{\sum_{i}\left|\partial_{i} b_{i}\right|}$

To analyze this error in details we visualize the threedimensional distribution of $\mathcal{E}$ within the whole domain at several times in all runs, and we calculate both the maximum value and standard deviation (i.e. the "rms value") of $\mathcal{E}$ considering different regions in space.

We find that, if the regions near all boundaries are excluded, the error calculated in several horizontal planes (at fixed $z$ ) globally remains constant at all times, with $\mathcal{E}^{\max }(z) \in$ [0.01; 0.2] and $\mathcal{E}^{\mathrm{rms}}(z) \in[0.0006 ; 0.003]$. The maxima are always located in a few gridpoints only for a given altitude, as suggested by the lower rms values. These same errors are in fact already present at $t=0$, due to the linear interpolations which were used to save the results of the potential field extrapolation in a non-uniform mesh (see Sect. 3.1). Including the regions near the open boundaries results in local enhancement of the errors typically by one order of magnitude. The amplitudes of these errors are also stable in time. A selection of the regions where the magnetic fields are the highest in the domain, i.e. for $(x ; y) \in[-30 \mathrm{Mm} ; 30 \mathrm{Mm}]$, but excluding the lower and the top boundaries in $z$, result in errors which are typically twice as low as for the whole box excluding the boundaries.

The most important errors are located near the lower linetied boundary at $z=0$. They are already present at $t=0$ on two grid layers along $z$, due to the magnetic field extrapolation in the ghost cells (see Eq. (34)). They increase with time at $z=0$ by a factor 2 , while they slowly propagate inside the domain as the twist increases. This propagation of the errors occurs at typical speeds of 5-10 $\mathrm{km} \mathrm{s}^{-1}$ which are much smaller than the velocities of Alfvén waves and of the expanding flux tube, and which are also slower than the photospheric driving velocities. For the twists considered in this paper, the errors always remains confined to $z<10 \mathrm{Mm}$. In this layer, $\mathcal{E}^{\max }(z)$ $\left(\mathcal{E}^{\text {rms }}(z)\right.$ respectively) decrease exponentially with $z$ from 1 (respectively 0.3 ) to their values inside the domain given above.

These numbers show that, even though the numerical scheme does not ensure $\boldsymbol{\nabla} \cdot \boldsymbol{b}=0$, the errors generated within the volume and the open boundaries are acceptable since they typically do not increase from their weak values at $t=0$. However, even though the line-tied boundary apparently does not result in a boundary layer when various fields are considered, it still generates non-negligible errors in $\boldsymbol{\nabla} \cdot \boldsymbol{b}$, which tend to propagate away from the boundary. Unfortunately, at that stage it is difficult to estimate the importance of these errors in the results of the calculations.

\section{Calculation of the equilibrium curve}

\subsection{Relaxation behaviour}

As pointed out in Sect. 4.3, the development of the dynamic phase in the continuously driven runs does not necessarily indicate the absence of equilibrium for a given twist. However, it does not show either that the system would always find an equilibrium if the driving velocities were smaller, since the expansion velocities become more than one order of magnitude larger than the driving velocities.

The motivations for analyzing the equilibrium properties of our twisted flux tubes are the following. Firstly, relaxations runs calculated from the continuously driven run shown in Amari et al. (1996) appear to be longer and more difficult to achieve as the flux tube enters the dynamic phase (Amari 2004, private communication). The same difficulty arises in magnetofrictional calculations of force-free twisted flux tubes, as shown by Klimchuk et al. (2000). Secondly, Török \& Kliem (2003) found a loss of equilibrium in one of their models (the one defined by their parameter $y_{0}=1$ ) for $\mathcal{N}>1.38-1.48$ turns. This loss of equilibrium, which they attributed to a kink-like instability, appeared as the fast expansion of their core twisted flux tube in the absence of photospheric driving. Thirdly, the comparison between our runs (which have three different vortex sizes) and those of Török \& Kliem (2003) (which have different initial bipolar potential fields) will consist in a parametric study which can lead to the finding of generic (non?) equilibrium properties of line-tied twisted flux tubes in general.

In order to search for the existence of equilibria from our continuously driven runs, we perform relaxation calculations following the method described in Sect. 3.3. Figure 6 shows the typical behaviour of a few relaxations of twisted flux tubes for various $(\mathcal{N} ; \zeta)$. For all runs, during the relaxation phase, the magnetic energy monotonically decreases by a few percent. A part of the lost magnetic energy is converted into kinetic energy. The latter is due to the velocities which are generated by the Lorentz forces present in the system at the start of the relaxations.

For some relaxation runs the vertical expansion velocities reached a few hundred $\mathrm{km} \mathrm{s}^{-1}$, which is not negligible compared to the Alfvén speed. In spite of this, we always find that the velocities decrease after a time of 50-300 s, and that they eventually become negative shortly after (see Fig. 6). So the flux tube starts to shrink back to lower altitudes in $z$ after its initial expansion. This behaviour is found for every relaxation run performed in this study, regardless of the initial altitude of the axial field line, i.e. of its distance to the top boundary. We checked that the vertical deceleration was always associated with a change in sign from positive to negative of the 

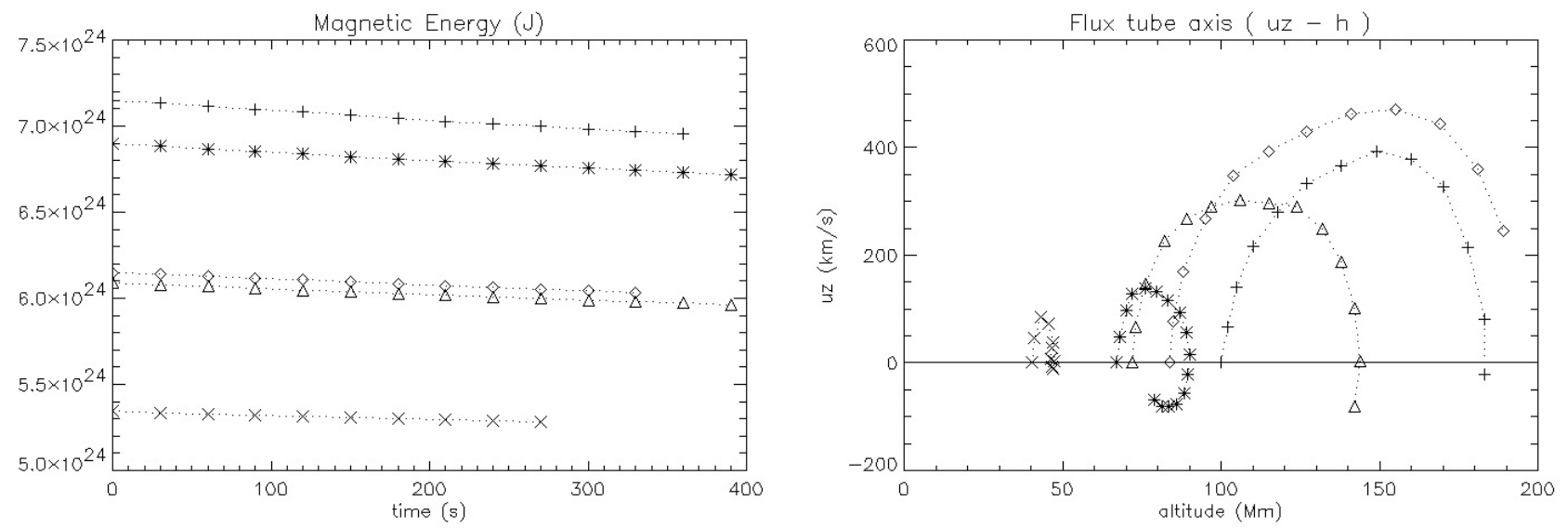

Fig. 6. Five examples of relaxation runs. Pluses correspond to $(\mathcal{N}=1.05 ; \zeta=10)$, stars to $(\mathcal{N}=0.92 ; \zeta=10)$, diamonds to $(\mathcal{N}=1.48 ; \zeta=0.9)$, triangles to $(\mathcal{N}=1.41 ; \zeta=0.9)$ and crosses to $(\mathcal{N}=1.66 ; \zeta=0.5)$. Each mark is separated in time by $\Delta t=30 \mathrm{~s}$. (Left): Magnetic energy as a function of time. (Right): Altitude-velocity diagrams showing the begining of damped oscillations along $z$.

vertical component of the Lorentz forces, which eliminates a pure diffusive origin. Also, performing relaxations with increased resistivity by a factor 3 has not led to qualititative changes.

So we conclude that the system always enters an oscillatory behaviour along a potential well around an equilibrium position. These oscillations result from the initial position of the flux tube away from the equilibrium point at the start of the relaxation, so that inertia allows the system to pass beyond this point, which is followed by the action of restoring forces which pull back the system toward the equilibrium. The existence of these oscillations obviously shows that the system is not overdiffusive, in spite of the strong value of $u_{v^{\star}}$ which we chose in Sect. 3.4.

Comparing various relaxation runs, we find that the initial vertical acceleration, the peak vertical velocities and the amplitude in $z$ of the oscillations for a given vortex size are all larger as the relaxation is performed for higher and higher twists $\mathcal{N}$. This indicates that the system proceeds further and further away from its equilibrium position as $\mathcal{N}$ increases during the dynamic phase of the continuously driven runs. However, our calculations suggest that the system can always find an equilibrium.

\subsection{Comparison with other authors}

The rapid increase of expansion rate of the equilibria as function of footpoint displacement that we find is consistent with other numerical calculations of twisted bipoles in Cartesian geometry by Amari et al. (1996), Klimchuk et al. (2000) and Török \& Kliem (2003), as well as with the behaviour of axisymmetric sheared dipoles in a spherical geometry (Roumeliotis et al. 1994). Our relaxation behaviour is consistent with Amari's qualitative statements and with the results of Klimchuk et al. (2000), about the increasing difficulty in relaxing the system for increasing twists. However, contrary to Török \& Kliem (2003), we find no evidence for a loss of equilibrium in any of our systems, even though their bipolar field resized to our scale results in a loss of equilibrium when the altitude their axial field line is less than $\sim 40 \mathrm{Mm}$, which is much smaller than the vertical extension of our domain.

This quantitative difference may be due to different numerical prescriptions between codes. Firstly, Török \& Kliem (2003) apparently use a much stronger diffusion term for momentum (and for density) than we do, since their "successful relaxation" runs apparently do not show oscillatory behaviour. Secondly, they use a non-uniform grid which is typically 3.3 times more stretched in $z$ than ours for similar altitudes, if their spatial units are rescaled to ours to have the same bipole size at $z=0$ (Törok 2004 , private communication). Thirdly, they use a different spatial scheme than we do. Fourthly, the development and consequences of non-zero $\boldsymbol{\nabla} \cdot \boldsymbol{b}$ in their runs must be different than in ours. Indeed, they use the standard conservative form of the MHD equations. Finally, their boundary conditions at $z=0$ on $\boldsymbol{b}$ are not the same as ours, even though their $b_{z}(z=0)$ varies only slowly with time. All these issues may result in differences in the calculation of spatial derivatives, hence of Lorentz forces.

The analysis in our relaxation runs of each term that contributes to the vertical acceleration in Eq. (9) shows that, around the time at which we find a deceleration, and around (and above) the altitude of the axial field line, the vertical derivative term $b_{y} \partial_{z} b_{y}$ is well resolved, and is at most 10 times larger than the total vertical component of the Lorentz force. A small difference in the calculation of this term, which may be amplified in time, may result in a different sign for the vertical Lorentz force. This issue will have to be addressed by new calculations in the future, if possible with different codes.

\subsection{Analytical equilibrium curve}

In order to derive the precise numerical equilibrium curves for our three vortex sizes, we need in principle to perform prohibitively long relaxation runs so as to follow the damping of the vertical oscillations of the magnetic field. We rather choose a simplified method. We measure the altitude in $z$ of the axial field line at the time for which the vertical acceleration first becomes negative during the relaxations (see Fig. 6). Since this 

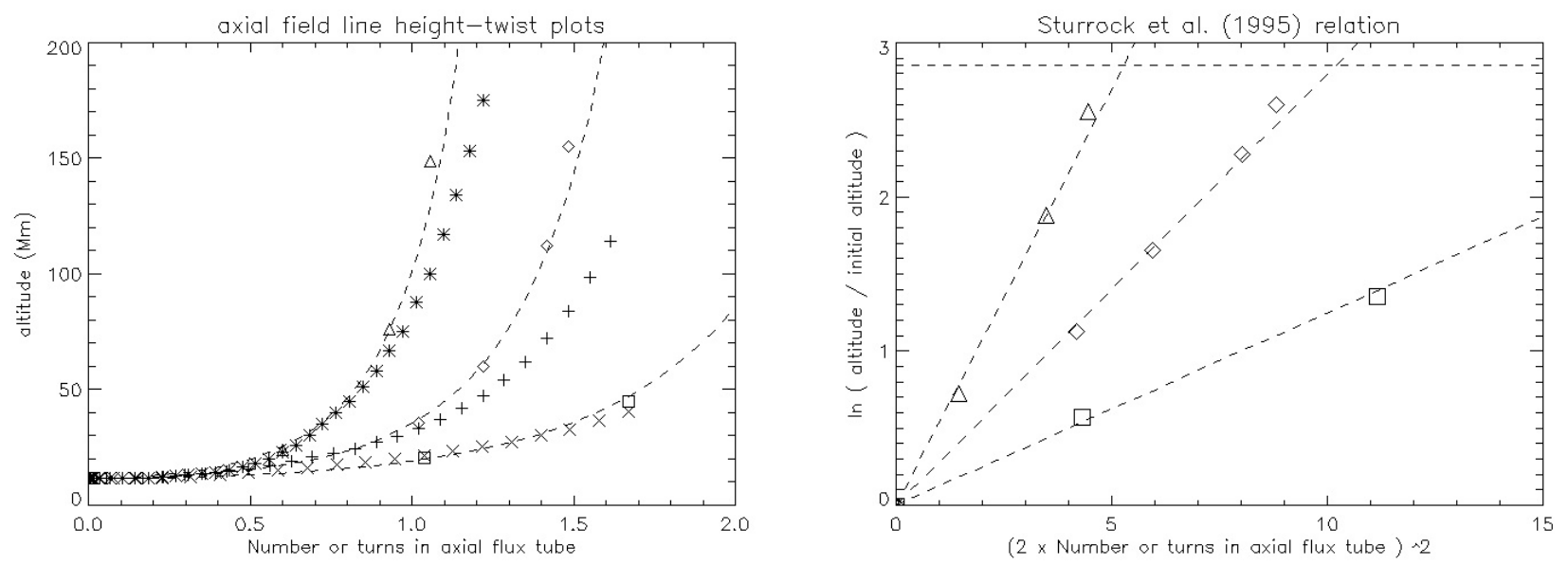

Fig. 7. Equilibrium curves derived from relaxation runs. (Left): Same as Fig. 4, bottom left, overlaid with [triangles; diamonds; squares] which correspond to "equilibrium" positions found from relaxation runs with $\zeta=[10 ; 0.9 ; 0.5]$. The corresponding equilibrium curves are overplotted as dashed lines. (Right): Same equilibrium curves and calculated "equilibrium" positions plotted with $\log / \mathrm{square}$ axes. The dashed horizontal line represents the height of the top boundary.

time is well matched in our runs by the change in sign of the vertical component of the Lorentz force, we then assume that this altitude corresponds to the asymptotic equilibrium of the system. The measured positions for various values of $(\mathcal{N} ; \zeta)$ are marked in Fig. 7, left on top of the height time plots from the continuously twisted runs. The equilibrium positions for a given twist are always located above those found when the system is continuously twisted. As conjectured in Sect. 4.3, this can be explained by the fact that the currents continuously generated by the vortices at $z=0$ do not have time to distribute all along the expanding field lines by Alfvén waves, especially during the dynamic phase during which the expansion rate is comparable to the Alfvén speed.

We find that these positions are well aligned in Fig. 7, right, in accordance with the "successful relaxation" plots of Török \& Kliem (2003) which were calculated for different physical conditions with a different code (recall in particular our approximate relaxation method and our problems with the $\boldsymbol{\nabla} \cdot \boldsymbol{b}$ constraint). For comparison, the three curves which link the marks calculated for all three $\zeta$ values are overplotted onto Fig. 7, left. So we find that all our equilibrium curves follow the analytical expression:

$h(\mathcal{N})=h_{\circ} \exp \left(\mathcal{A} \mathcal{N}^{2}\right)$,

where $h$ is the altitude of the axial field line at a given twist $\mathcal{N}$, $h_{\circ}$ is the altitude of this field line for the potential field and $\mathcal{A}$ is a geometrical factor which, for a fixed initial potential field, depends on $\zeta$ i.e. on the vortex sizes.

Our numerical reconstructions of these curves show that for $\zeta=(10 ; 0.9 ; 0.5), \mathcal{A} \simeq(2.16 ; 1.12 ; 0.50)$. By comparison, Török $\&$ Kliem (2003) find $\mathcal{A} \simeq 0.75$ before their flux tube loses its equilibrium. This value lies within the range of $\mathcal{A}$ which we found. Also it is now clear that our magnetic configuration always enters an oscillatory stage during its relaxation, even when the equilibrium curve is steeper than the one of Török \& Kliem (2003). Both these issues suggest a posteriori that their loss of equilibrium is not be physical, and that simple bipolar twisted flux tubes can always evolve quasistatically if the photospheric driving velocities are infinitesimal.

As noted by Török \& Kliem (2003), the analytical equilibrium curve given in Eq. (42) also applies to other geometries, specifically to spherical axisymmetric (2.5D) dipoles subject to azimuthal shearing motions (Roumeliotis et al. 1994; Sturrock et al. 1995). The derivation of this curve by both analytical and numerical means, by several authors and in various geometrical conditions, implies that it is a generic property of line-tied force-free fields, both sheared and twisted.

\section{Observational properties}

\subsection{Coronal mass ejections}

In the following we address the question of the applicability of the continuously twisting bipolar flux tube model to the coronal mass ejection (CME) phenomenon. We assume that the analytical expression of the equilibrium curve (Eq. (42)) remains valid with altitude until the apex of the axial field line reaches the top of helmet streamers, where the solar wind becomes dominant (i.e. $2-3 R_{\odot}$ ). We also assume a photospheric twisting rate $\mathcal{N}(t)=u_{\text {phot }} /\left(2 \pi R_{\text {phot }}\right)$, where $u_{\text {phot }}$ is the twisting velocity and $R_{\text {phot }}$ is the radius of the small region which defines the twist within each vortex $\left(R_{\text {phot }}=1.5 \mathrm{Mm}\right.$ for $h_{\circ}=11.5 \mathrm{Mm}$, see Sect. 4.1). Equation (42) then leads to:

$$
\begin{aligned}
& h(t)=h_{\circ} \exp \left[\mathcal{A}\left(\frac{u_{\mathrm{phot}} t}{2 \pi R_{\mathrm{phot}}}\right)^{2}\right], \\
& v_{z}(t)=2 \mathcal{A} t\left(\frac{u_{\mathrm{phot}}}{2 \pi R_{\text {phot }}}\right)^{2} h(t) .
\end{aligned}
$$

These analytical expressions should in principle be recovered numerically in the limit of infinitesimal photospheric twisting velocities. Figure 8 shows some corresponding plots using two values of $\left(h_{\circ} ; R_{\text {phot }}\right)$ scaled by the same factor $f$ (one typical of 

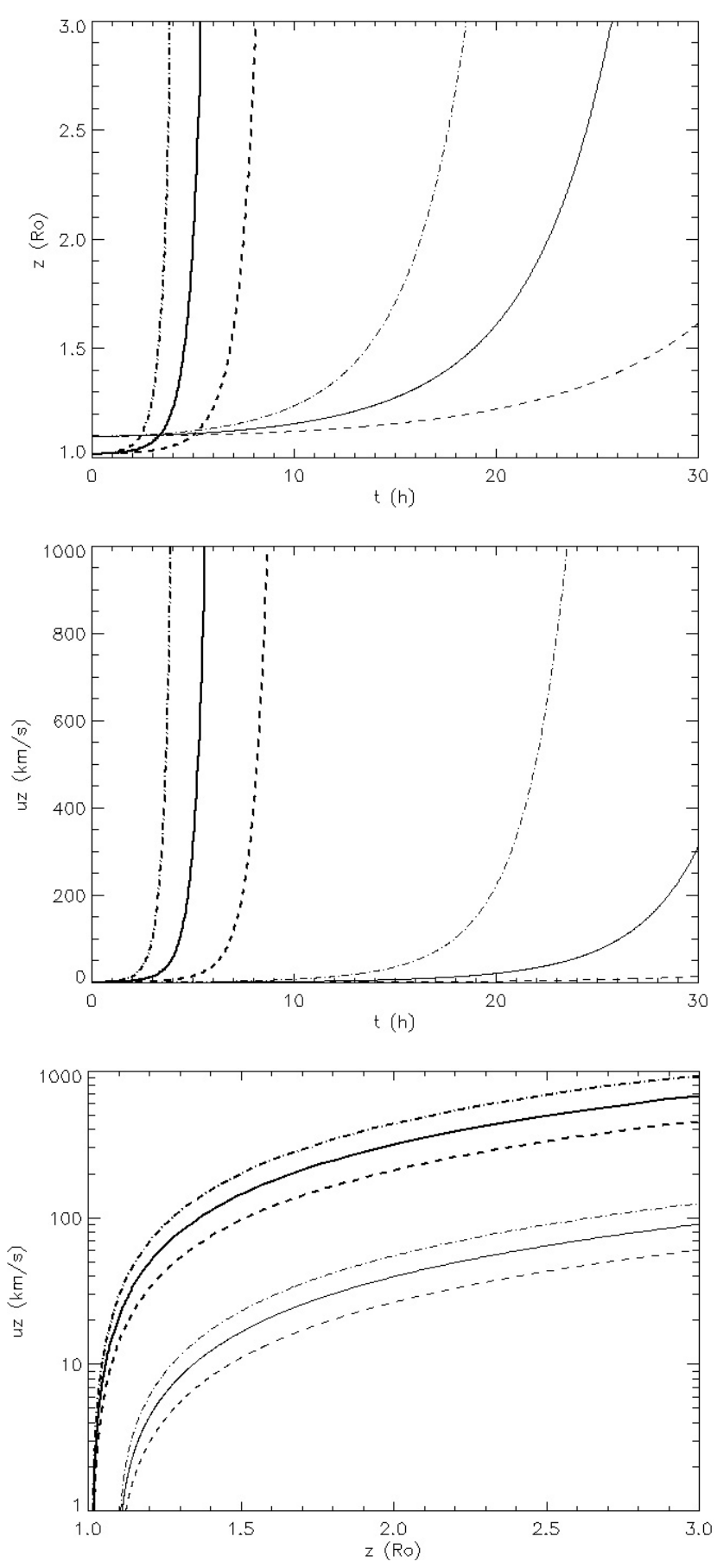

Fig. 8. Simulated height-velocity-time plots of the axial field line of continuously driven flux tubes twisted with $u_{\text {phot }}=1 \mathrm{~km} \mathrm{~s}^{-1}$, for altitudes between the photosphere $\left(z=1 R_{\odot}\right)$ and the top of helmet streamers $\left(z=2-3 R_{\odot}\right)$. [thick; thin $]$ lines correspond to $h_{\circ}=[11.5 ; 69] \mathrm{Mm}$ and to $R_{\text {phot }}[1.5 ; 9] \mathrm{Mm}$. [dash-dotted; continuous; dashed $]$ lines correspond to $\zeta=[10 ; 0.9 ; 0.5]$.

the size a young active region and another typical of a prominence) and three values of $\mathcal{A}$ (corresponding to the three vortex sizes calculated in this paper).

Regardless of the velocity magnitudes, Fig. 8 illustrates one typical property of Eq. (42): the scale dependence. It is clear that the system size (i.e. prominence as opposed to active region) scales inversely with the acceleration and to the velocities. In particular, the velocity (resp. acceleration) at $h \gg h_{\circ}$ roughly scales like $f^{-1}$ (resp. $f^{-2}$ ). All these properties are consistent with the two classes of observed CMEs (Gosling et al. 1976; MacQueen \& Fisher 1983; Delannée et al. 2000; Andrews \& Howard 2001): the fastest (resp. slowest) ones mostly originate from active regions (resp. quiescent prominences), and once they reach the inner edge of the $\mathrm{SoHO} / \mathrm{LASCO}$ coronographs, they typically have radial velocities of the order of 500-1500 $\mathrm{km} \mathrm{s}^{-1}$ (resp. 50-300 $\mathrm{km} \mathrm{s}^{-1}$ ). As in the flux rope model of Chen \& Krall (2003) which considers end-to-end twists of about 4 turns (Chen 2004, private communication) the present explanation for both classes of CMEs is based on the size difference between prominences and active regions. However, contrary to Chen \& Krall (2003), our model does not involve the evolution of the flux tube curvature during a free expansion, but rather a direct scaling effect of the equilibrium curve during a driven expansion.

The velocity magnitudes in Fig. 8 have been calculated for a photospheric velocity of $1 \mathrm{~km} \mathrm{~s}^{-1}$ so as to obtain typical CME velocities at $3 R_{\odot}$. Even though the observed dynamics of CMEs have typically been studied at greater heights, a few height-velocity-time plots have been measured at lower altitudes (Srivastava et al. 1999, 2000; Wang et al. 2003; Zhang et al. 2004). Even though these observed plots appear to be in qualitative agreement with Fig. 8, two strong differences must be emphasized. Firstly, the prescribed $u_{\text {phot }}$ is at least ten times larger than photospheric velocities typically observable in rotating sunspots (Brown et al. 2003, and references therein). Secondly, the initial "quiet phase" before the "very fast opening" is not longer than a few hours (resp. a day) for the active region (resp. prominence) case. This is much too small compared to observed time-scales which are of the order of days to weeks.

The only way to bypass the first difficulty (too fast photospheric flows) would be to have twist either injected by reconnection rather than by flows, or by fast undetectable flows within sunspots due e.g. to twisted flux emergence through the photosphere. The second issue (too short quiet phase) would imply that twist is not injected continuously, but rather in episodic bursts well related with the launch of CMEs, with time scales of the order of a few hours to one or two days. Direct application of this model to the CME phenomenon thus requires the same artificially fast twisting as in the analytical driven model of Chen (1996).

However, as emphasized by Forbes (2001), "this point of view is difficult to reconcile with the extremely tranquil conditions that exist in the photosphere during flares and CMEs". In our opinion, since the dynamic evolution of the weak fields surrounding the core twisted flux tubes plays a very important role in the dynamics of the system for twist values $\geq 1$ turn (see Sect. 4.4), their global reconfiguration associated with e.g. distant flux emergence (Lin et al. 2001) and/or magnetic reconnection (Antiochos et al. 1999) is most likely to lead to a CME. In the frame of our model, such reconfigurations may be associated with dynamic changes of the value of the parameter $\mathcal{A}$ in Eq. (42), which may solve the problem of the speed of the twist injection encountered by the present model and in Chen (1996). 

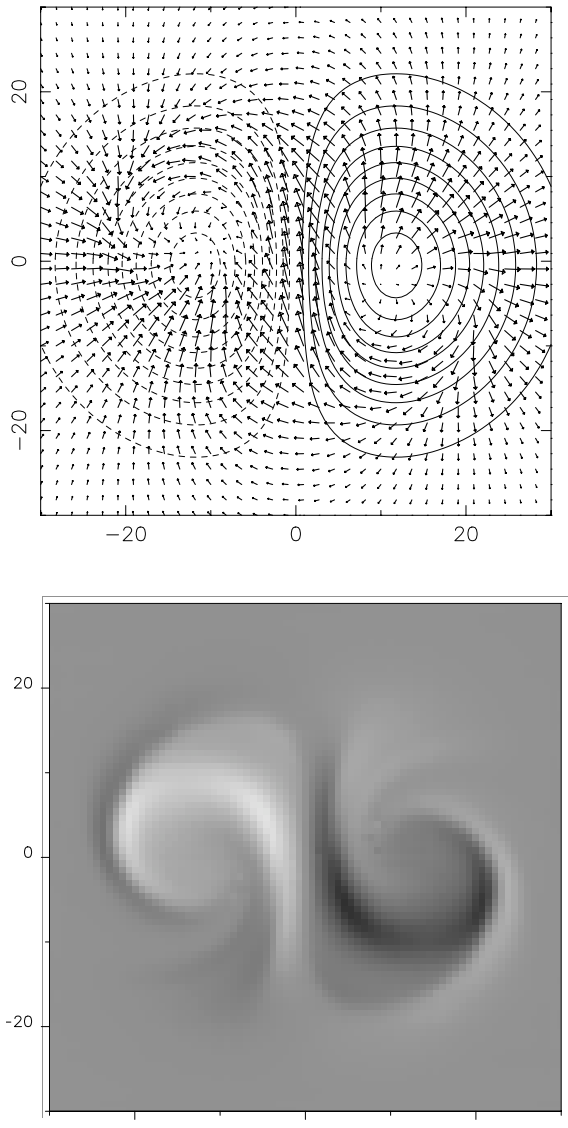

$-20$
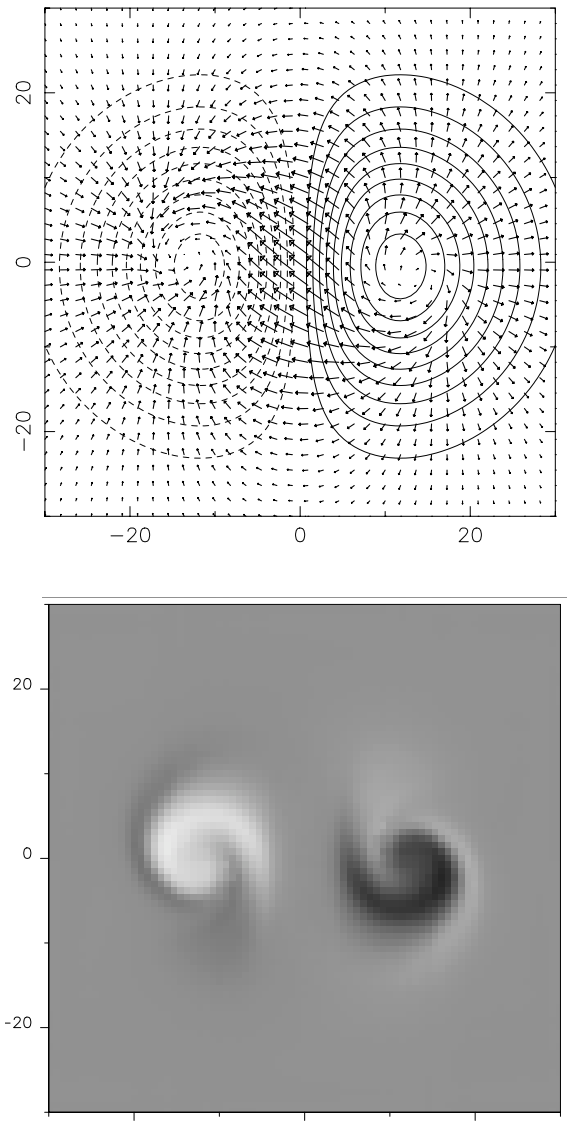

$-20$
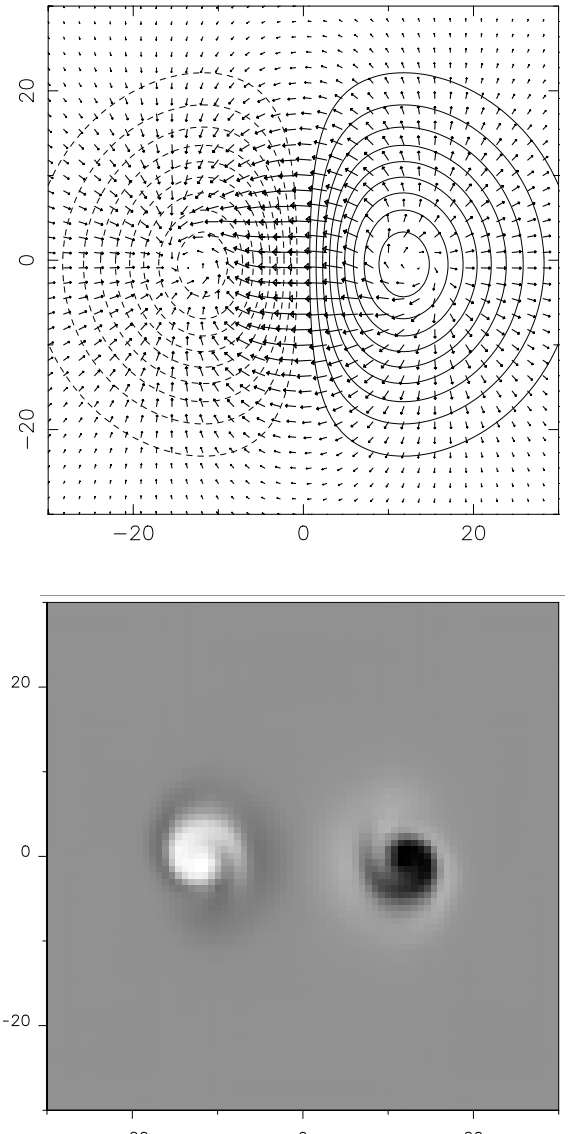

$-20$

Fig. 9. (First row): Contours of $b_{z}= \pm 50 ; 100 ; 150 ; 200 ; 250 ; 300 ; 350 ; 400 ; 450 \mathrm{G}$ and transverse field vectors at $z=0$. (Second row): Greyscale images of $j_{z}$ at $z=0$. White/black correspond to $j_{z}=9 \mathrm{~mA} \mathrm{~m}{ }^{-2}$. The [first; second; third] columns correspond to vortices defined by $\zeta=[10 ; 0.9 ; 0.5]$. All figures are plotted for a central twist $\mathcal{N} \sim 1.25$ turns.

\subsection{Photospheric electric currents}

We calculate the vertical electric current densities from the photospheric transverse fields,

$J_{z}=\mu^{-1}\left(\partial_{x} b_{y}-\partial_{y} b_{x}\right)$,

which result from the MHD evolution of the initial potential field subject to all three vortex sizes considered in this paper. Both are plotted in Fig. 9 for a central twist of $\mathcal{N} \sim 1.25$ turns. Some generic properties are found in all three cases, and are discussed below.

Within each magnetic polarity, both direct and surrounding return currents co-exist. The extended weak return currents naturally form by shearing/twisting an initial line-tied potential field, so as to maintain the outer untwisted fields potential. The direct currents are more concentrated and intense. Their maximal values are about $\left|J_{z}\right| \sim 6-9 \mathrm{~mA} \mathrm{~m}^{-2}$ for the largest and the smallest vortex sizes respectively. If the magnetic field amplitudes of the bipole were scaled to those of typical sunspots $\left(b_{z}^{\max }=2000 \mathrm{G}\right.$ instead of $\left.480 \mathrm{G}\right)$, these currents would be $\left|J_{z}\right| \sim 24-36 \mathrm{~mA} \mathrm{~m}^{-2}$, in accordance with typical vector field measurements within sunspots (see e.g. Pevtsov et al. 1995; Leka \& Skumanich 1999; Régnier et al. 2002).

These currents correspond to a negative value of the forcefree parameter $\alpha$ which is defined as $\alpha=\mu_{z} b_{z}^{-1}$. This sign is consistent with the prescribed boundary motions, which result in a left-handed magnetic twist, so a global negative magnetic helicity. For all three cases the maximum values are nearly the same: $\alpha \sim-0.25 \mathrm{Mm}^{-1}$. This is so because the more concentrated the vortex, the higher $J_{z}$ and the higher $b_{z}$ at the location of these strong currents (see Fig. 9). So the typical scale-length $L=2 \pi \alpha^{-1} \simeq 25 \mathrm{Mm}$ of the strongest current-carying field lines is the same for all three cases.

This $\alpha$ value is typically $15-30$ times larger than what is found in linear force-free field models of large-scale loops in sheared active regions (Schmieder et al. 1996; Démoulin et al. 2002). It is also 5-10 times larger than in models of intermediate and plage prominences (Aulanier \& Démoulin 2003) and X-ray sigmoïds (van Driel-Gesztelyi et al. 2000; Gibson et al. 2002). Note that in these past works, $\alpha$ was calculated for typically 2-8 times larger configurations than those studied here. So the present rescaled $\alpha$ values fall in the range of those estimated for prominences and sigmoïds.

Another generic property is a strong azimuthal asymmetry of the distribution of the transverse fields and of $J_{z}$ with respect to the vortex centers (compare Figs. 3 and 9). Consider one magnetic polarity, e.g. for $x<0$. Firstly, the apparent vortex centers as deduced from the transverse fields are always shifted from the real vortex centers located at $b_{z}^{\max }$. Secondly, the transverse fields originate radially from the vortex centers in a quadrant $\left(x<x_{\mathrm{c}} ; y<y_{\mathrm{c}}\right)$, where $\left(x_{\mathrm{c}} ; y_{\mathrm{c}}\right)$ are the coordinates of the 
left vortex. On the contrary, the transverse fields tend to follow the contours of $b_{z}$ for another quadrant $\left(x_{\mathrm{c}}<x<0 ; y>y_{\mathrm{c}}\right)$. Thirdly, $J_{z}$ shows a swirling pattern around $\left(x_{\mathrm{c}} ; y_{\mathrm{c}}\right)$. Several spiral arms of opposite signs for $j_{z}$ and of various widths appear for $\mathcal{N}>0.5$ turn, and develop more and more with time as the twist increases.

This azimuthal asymmetry is not present in cylindrical MHD calculations in which both extremities of the cylinder are line-tied. It is a geometrial property caused by the curvature of the field lines which are line-tied onto the same plane. This asymmetry comes from the fact that, even though the same amount of twist $\Phi$ is injected for both short field lines (rooted on each side of the inversion line around $x=0$ ) and large ones (located on the edges of the bipole), stronger currents are generated in the smaller field lines. This can be explained by considering a simple cylindrical flux tube model in $(r ; z ; \phi)$, where $l$ is the length of the flux tube along $z$. The electric current and field line equations in cylindrical coordinates result in:

$J_{z}=r^{-1} \partial_{r}\left(r b_{\phi}\right) \propto b_{\phi}$

$b_{\phi}=r l^{-1} b_{z} \Phi$.

It naturally follows that for $(r, \Phi)$ fixed, shorter field lines (having small $l$ ) will contain stronger currents since $J_{z} \propto l^{-1}$. As the twist proceeds, relatively stronger currents are generated near the footpoints of the initially shorter field lines, resulting in an asymmetry not only in $x$, but also in $y$. This effect is even more amplified by the expansion of the large field lines with increasing twist (including the core twisted flux tube): this expansion is faster than the expansion of the smaller field lines. This amplification is then partly responsible for the gradual inclusion of the weak return current region within the swirling pattern of $J z$ described above, around the footpoints of the fast expanding core twisted flux tube.

Interestingly, the complex asymmetric structure of $J_{z}$ in the photosphere is formed within a very simple magnetic configuration which is initially smooth, bipolar and symmetric, and which is twisted by vortex motions that are also very simple. Also, this structure qualitatively remains very similar for extended as well as concentrated vortices. So it is probably a generic property of line-tied twisted flux tubes rooted in the photosphere. It follows that twisted sunspots should naturally have - at least - two more-or-less contrasted concentrations of strong direct electric currents within a given magnetic polarity, and possibly - at least - one patch of weak return current of opposite sign. This is in very good agreement with the observations (Pevtsov et al. 1995; Leka \& Skumanich 1999; Régnier et al. 2002). In the frame of our model, the observed fragmentation of the force-free parameter $\alpha$ within sunspots is then not necessarily due to some hidden complexity in the distribution of the photospheric flux or of the footpoint twist.

\subsection{X-ray sigmoïds}

A common assumption for the materialization of observed solar structures is that they are directly traced by magnetic field lines. However, it has already been shown for the case of filaments and prominences that this assumption is not fully valid, and that only portions of field lines trace the visible features, both in $\mathrm{H} \alpha$ and in EUV wavelengths (e.g. Aulanier \& Schmieder 2002). In the following we address the validity of this assumption for sigmoïdal structures observed in X-rays. Our main hypothesis is that since $\mathrm{X}$-rays make it possible to visualize the hottest parts of the corona, the observed intensity $I_{\mathrm{X} \text {-rays }}$ is nearly proportional to the Joule heating term integrated along the line of sight. If we consider the bipole to be at the disc center for simplicity, we simulate X-ray observations by calculating:

$I_{\mathrm{X} \text {-rays }}(x ; y) \propto \int \boldsymbol{J}^{2}(x ; y ; z) \mathrm{d} z$.

This assumption is very approximate since the energy equation, whose proper treatment would imply very short timescales, is not integrated in our present calculations. In particular, the issue of thermal conduction along heated field lines, which can result in their filling by dense material originating from chromospheric evaporation, is not considered. Keeping this limitation in mind, we analyze the results of the simulated $\mathrm{X}$-ray observations for all three vortices at the same twist $\mathcal{N} \sim 1.25$ turns.

Figure 10, top shows that sigmoïdal structures of various shapes naturally appear for all three cases, with an inverse$\mathrm{S}$ shape. Their orientation is compatible with the sign of the force-free parameter $\alpha$ found above and with the hemispheric rule for observed sigmoïds and magnetic helicity, i.e. $\alpha<0$ and inverse-S shapes in the northern hemisphere (Seehafer 1990; Pevtsov et al. 1995; Rust \& Kumar 1996; Burnette et al. 2004). The sigmoïd formed by large vortices is the brightest of all three, and its brightest region is right above the inversion line. On the contrary, the sigmoïd formed by small vortices has its brighests regions located at its extremities, on top of the center of each vortex. The sigmoïd formed by medium vortices is an intermediate case, where the brightest regions are located at the elbows of the S-shape.

A global idea for the altitude of current formation responsible for the sigmoïds and for their different shapes as a function of vortex size can be obtained as follows: on one hand some portions of the sigmoïds are well matched by the distribution of $J_{z}(z=0)$ (compare Figs. 9 and 10). This shows that they correspond to low altitude features. For a given twist, these regions are brighter for smaller vortices because the core twisted flux tube is more confined at low altitude than for extended vortices (as shown by the equilibrium curves, see Fig. 7), which results in stronger electric currents (see Eqs. (46) and (47)). On the other hand some other portions of the sigmoïds cross the inversion line at $x=0$. These originate from an emitting volume located substantially above the photosphere. These regions are brighter for larger vortices because their extension results in a stronger shearing of the field lines located near the inversion line at $x=0$ (as shown e.g. by transverse fields in Fig. 9).

The three-dimensional nature of the sigmoïds is revealed by the integration of field lines whose photospheric footpoints (at $z=0$ ) are located all along the projected bright sigmoïds. Generic results are found for all three cases (see Fig. 10, second $\&$ third rows). 

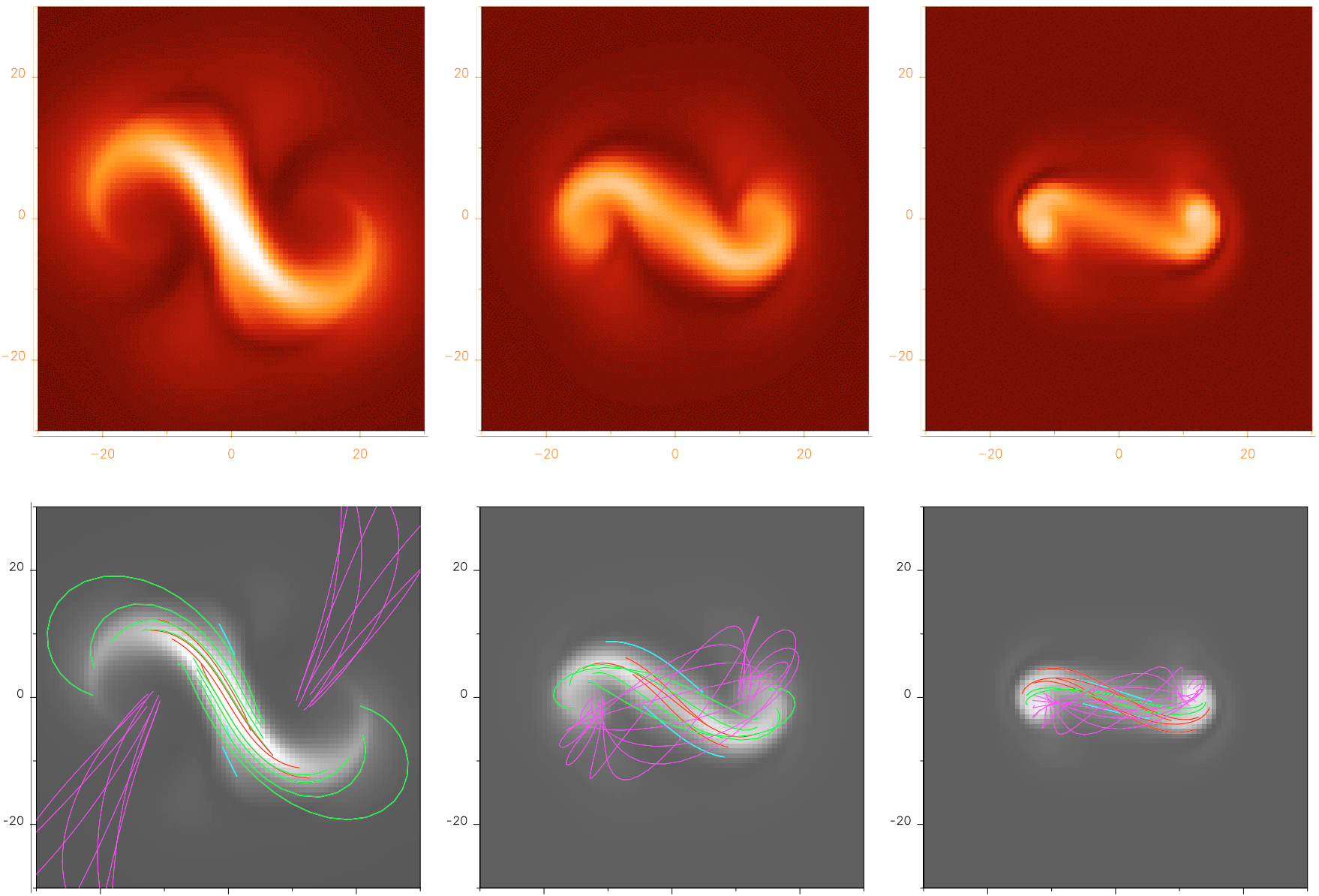

$-20$

20

$-20$

0

$-20$

20
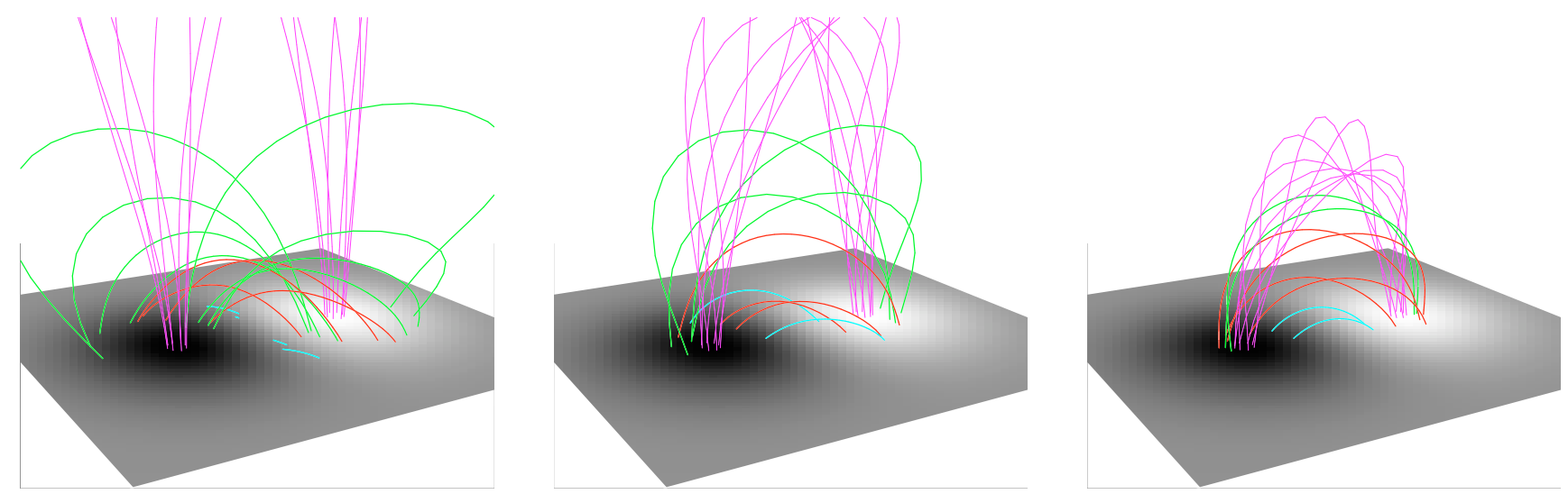

Fig. 10. (First row): X-ray sigmoïds calculated from the vertical integration of the Joule heating term. The minimal (black) and maximal (white) intensities are scaled to the same values for all three cases. (Second row): Sigmoïd with field lines overplotted. [Pink; green; red; blue] lines are rooted respectively around [the vortex centers; the sigmoïd ends; their brightest parts; their edges]. (Third row): Projection view of the same field lines with greyscale for $b_{z}$ at $z=0$. The [first; second; third] columns correspond to vortices defined by $\zeta=[10 ; 0.9 ; 0.5]$. All figures are plotted for a central twist $\mathcal{N} \sim 1.25$ turns.

First, it is evident that the sigmoïds are not traced by the core twisted flux tube which is rooted around the vortex centers (shown as pink field lines): the deformation of this flux tube by the antisymmetric generation of currents forms in fact a direct$\mathrm{S}$ shape, opposite to the orientation of the sigmoïd. Also, one can see that the sigmoïd is always present, regardless of the vertical expansion of the flux tube. Indeed its apex almost reaches the top of the numerical box for the large vortex case at the time of the figure.

Actually, we find that the sigmoïds are traced by an ensemble of sheared (or weakly twisted) field lines located at low altitudes beneath the twisted flux tube, whose deformation from their initial potential configuration is directly related to the direction of the photospheric vortices. 
But, when one attempts to match a sigmoïd by a unique field line from this ensemble, one sees that it is impossible. In reality, no single field line can trace the whole of the sigmoïds, even though a few ones which are nearly symmetric with respect to the inversion line can trace the central parts of the sigmoïds. This is mostly noticeable for the sigmoïd ends (especially for the large vortex case), where the field lines projected onto the $z=0$ plane rarely trace the shape of the simulated sigmoïds. This result, if true in general, implies that trying to match observed sigmoïdal structures directly with calculated field lines is impossible.

Projection views (Fig. 10, third rows) show that the apex of the field lines which are rooted on top of the brightest parts of the projected sigmoïds (shown in red) and at their ends (shown in green) nearly has the same altitude for all three vortex sizes for $\mathcal{N} \sim 1.25$ turns. This may look surprising at first sight for two reasons. First, this behaviour is very different from that of the core twisted flux tube, whose vertical expansion very strongly depends on the vortex size. Second, the field lines which form the sigmoïds are rooted in different regions for different vortex sizes. However, this property is related to the fact that both $\alpha$ (so the length-scale of the currents $L=2 \pi \alpha^{-1} \simeq$ $25 \mathrm{Mm}$ ) and the distances between the footpoints of these field lines are nearly the same for all cases. This leads to the prediction that the altitude of current-carrying field lines which form the sigmoïds for a given photospheric magnetic field does not depend on the width of the current-generating vortex, but only on the degree of twist of the strongest fields.

The same analysis has been performed for lower and higher degrees of twist (always before the flux tube reaches the top boundary) for all three vortex classes. Apart from qualitative morphological differences, the same properties were found in every case. This leads to the conclusion that, under the hypothesis which we used to simulate $\mathrm{X}$-ray observations, our findings are generic.

It is worth noting that the properties that we find (i.e. sigmoïds being formed by Joule heating in an ensemble of sheared field lines located beneath a twisted flux tube and all rooted in the photosphere within the direct currents) are contradictory with the results of the non-linear force-free field model of Régnier et al. (2002). They associated an observed sigmoïd both with a strongly twisted flux tube and with a less twisted one, each having a different sign for $\alpha$. This contradiction may either be attributed to the unreliability of our proxy (see Eq. (48)) for simulating X-ray observations, or to the fact that the X-ray loops analyzed by Régnier et al. (2002) show a rather weak S-shape, so that they might not be a real sigmoïd. This point will have to be addressed again in the future.

\section{Conclusion}

In this paper we calculated the structure and evolution of slowly twisted flux tubes in the solar photosphere and in its corona, using a new zero- $\beta$ MHD code with line-tied boundary conditions at the photosphere and open boundary conditions at the other faces. We considered three different vortex sizes which preserve $b_{z}$ at the photospheric boundary. Our domain was $\sim 20$ times higher than the initial altitude of the field line which is rooted in the strongest field regions. We compared our results with previously published calculations of similar configurations. Our parametric study resulted in the finding of generic properties in such systems.

We have shown that continuously twisted and relaxing flux tubes all have the following generic properties:

(1) No evidence of instability: Twisted flux tubes always reach a non-linear force-free equilibrium if the driving velocities are suppressed. There is no evidence for instability or a loss of equilibrium. Numerically, we find that the relaxation from continuously driven runs results in damped oscillations of the system around an equilibrium position, whose amplitude increases with the twist.

(2) Generic altitude-twist relation: The equilibrium curve as a function of twist is given by Eq. (42), which surprisingly is the same analytical formula as for axisymmetric spherical sheared dipoles. The steepness of this equilibrium curve for twists larger than 0.7-1.5 turns is responsible for a change of behaviour in continuously twisted runs, from a quasistatic phase to a dynamic phase. This transition is characterized by the appearence of fast expanding motions, in addition to the low-amplitude torsional Alfvén waves generated by the photospheric vortices. However, this transition is not an instability in the standard sense, since it requires continuous driving to develop.

(3) Influence of the outer fields: The steepness of the equilibrium curve, the vertical expansion of the twisted flux tube at a given twist and the time at which the dynamic phase begins all strongly depend on the extension of the photospheric vortices. This shows that the dynamics of the strong field regions are very sensitive to the evolution of the outer field lines rooted in weak field regions. This is consistent with some recent 2.5D MHD models of solar eruptions: (i) the breakout model of Antiochos et al. (1999) where a quadrupolar topology can result in a resistive eruption; and (ii) the analytical flux emergence models of Lin et al. (2001), which result in complex equilibrium curves and critical points as a function of the model parameters. Note that our stability properties have yet only be shown for bipolar configurations having a continuous distribution of the vertical component of the magnetic field at the line-tied boundary. In other words, the configurations that we considered always incorporate some large-scale untwisted flux. Based on our large vortex calculations, in which $\sim 85 \%$ of the magnetic flux is twisted, and for which the core twisted flux tube is the most expanded at a given twist, we would still argue that fully twisted discrete flux tubes still will not become unstable. Our argument is based on the fact that for a given end-to-end twist $\mathcal{N}=1+\epsilon$ turns, the more photospheric flux is twisted (by increasing the vortex size), the larger the vertical expansion (hence the length) of the core twisted flux tube, so the smaller the twist per unit length (as well as the field aligned currents). Furthermore according to Baty (2001) the twist per unit length is a more relevant quantity for defining the onset of the kink instability than the total end-to-end twist. So we would argue that the more flux is twisted, the less the system will be subject to the 
kink instability. Nevertheless, field opening may be caused by other processes than the kink, e.g. a loss of equilibrium, but our present calculations do not make it possible to address this issue since we always find an equilibrium.

Even though the initial potential field is symmetric, bipolar, smooth, and twisted by simple photospheric vortices, the following complex observational properties of the Sun's atmosphere can naturally be explained for various vortex sizes at various degrees of twist:

(1) CME dynamics: The steepness of the equilibrium curves extrapolated to $3 R_{\odot}$ combined with twisting velocities of $1 \mathrm{~km} \mathrm{~s}^{-1}$ permits us to reproduce velocity-height-times plots at low altitudes for both fast and slow coronal mass ejections (CMEs). In this model, the difference between two kinds of CME is only due to the scaling of Eq. (42), i.e. to the respective sizes of active regions (AR) and quiescent prominences. However, it is not possible to use this model as it is for CMEs, because the required photospheric velocities (about ten times larger than observed) and the relatively short time-scale for quasi-static expansion phases (from a few hours for AR-related CMEs to $\sim 1$ day for prominence-related CMEs) are contradictory with observations. Building an acceptable CME model based on continuous slow photospheric driving is nevertheless possible. It would require the connection of two equilibrium curves by e.g. distant reconnexions in outer field lines, since the steepness of the equilibrium curve strongly depends on the evolution of outer field lines rooted in weak field regions. This issue is to be examined in a later work.

(2) Photospheric currents: Both shear and twist are traceable from the transverse components of the magnetic field in the photosphere. There the vertical currents show complex swirling patterns which result in asymmetric concentrations within each magnetic polarity, surrounded by diffuse return currents. This is in accordance with typical vector field measurements within sunspots in the photosphere. The complex patterns are due both to the simultaneous twisting of both short and long field lines, resulting in different current generation, and to the fast expansion of the most twisted field lines around the vortex centers. For larger (resp. smaller) vortices, the strongest currents are located in weaker (resp. stronger) vertical fields in the photosphere, and are mostly associated with sheared (resp. twisted) field lines. For equal central twists, the maximum value of the force-free parameter $\alpha$ is nearly the same for various vortex sizes. Both simulated current magnitudes and $\alpha$ values are consistent with observations.

(3) X-ray sigmoids: Sigmoïdal structures calculated from the vertical integration of the Joule heating term naturally form above the strongest photospheric currents. Their ends (resp. central parts) are brighter for concentrated (resp. extended) photospheric vortices. Their orientation is opposite to the deformed central twisted flux tube. It is consistent with the sign of $\alpha$ and with solar hemispheric rules for currents and X-ray sigmoïds ( $\alpha<0$ and inverse-S shapes for the northern hemisphere). No single field line can ever trace the whole shape of the simulated sigmoïds. The ensemble of field lines which pass through the sigmoïds is sheared (or moderately twisted) and relatively low-lying, located below the central twisted flux tube. Projected onto the photospheric plane, sigmoïd ends are composed of J-shaped field lines, and their centers of weakly S-shaped ones. For equal central twists, regardless of the vortex sizes, these J- and $\mathrm{S}$-shaped field lines have comparable altitudes and $\alpha$ values. If this model is correct, observed $\mathrm{S}$ shapes in X-rays are poor indicators of the magnetic twist. Viewed in oblique projections, the field lines which pass through the sigmoïds no longer show the J- or S-shapes. So this model readily explains the lack of sigmoïd observations near the solar limb.

Acknowledgements. G.A. thanks S. K. Antiochos and C. R. DeVore for very fruitful discussions about numerical methods, as well as T. Amari, B. Kliem and T. Törok for discussions about their work. The calculations in this paper were all done on the Digital-UNIX ES40 computer of the Service Informatique de l'Observatoire de Paris (SIO).

\section{References}

Amari, T., \& Luciani, J. F. 1999, ApJ, 515, L81

Amari, T., Luciani, J. F., Aly, J. J., \& Tagger, M. 1996, ApJ, 466, L39 Andrews, M. D., \& Howard, R. A. 2001, Space Sci. Rev., 95, 147

Antiochos, S. K., DeVore, C. R., \& Klimchuk, J. A. 1999, ApJ, 510, 485

Aulanier, G., \& Démoulin, P. 1998, A\&A, 329, 1125

Aulanier, G., \& Démoulin, P. 2003, A\&A, 402, 769

Aulanier, G., \& Schmieder, B. 2002, A\&A, 386, 1106

Baty, H. 2000, A\&A, 360, 345

Baty, H. 2001, A\&A, 367, 321

Brown, D. S., Nightingale, R. W., Alexander, D., et al. 2003, Sol. Phys., 216, 79

Burnette, A. B., Canfield, R. C., \& Pevtsov, A. A. 2004, ApJ, 606, 565

Chen, J. 1996, J. Geophys. Res., 101, 27499

Chen, J., \& Krall, J. 2003, J. Geophys. Res. (Space Physics), 108, SSH 2.1

Démoulin, P., Bagalá, L. G., Mandrini, C. H., Hénoux, J. C., \& Rovira, M. G. 1997, A\&A, 325, 305

Démoulin, P., Mandrini, C. H., van Driel-Gesztelyi, L., et al. 2002, A\&A, 382, 650

Delannée, C., Delaboudinière, J.-P., \& Lamy, P. 2000, A\&A, 355, 725

Forbes, T. G. 2001, AGU Spring Meeting Abstracts, 41

Gibson, S. E., Fletcher, L., Del Zanna, G., et al. 2002, ApJ, 574, 1021

Gosling, J. T., Hildner, E., MacQueen, R. M., et al. 1976, Sol. Phys., 48,389

Hood, A. W. 1992, Plasma Physics and Controlled Fusion, 34, 411

Hudson, H. S., Lemen, J. R., St. Cyr, O. C., Sterling, A. C., \& Webb, D. F. 1998, Geophys. Res. Lett., 25, 2481

Klimchuk, J. A., Antiochos, S. K., \& Norton, D. 2000, ApJ, 542, 504

Leka, K. D., Canfield, R. C., McClymont, A. N., \& van Driel-Gesztelyi, L. 1996, ApJ, 462, 547

Leka, K. D., \& Skumanich, A. 1999, Sol. Phys., 188, 3

Lin, J., Forbes, T. G., \& Isenberg, P. A. 2001, J. Geophys. Res., 106, 25053

MacQueen, R. M., \& Fisher, R. R. 1983, Sol. Phys., 89, 89 
Manoharan, P. K., van Driel-Gesztelyi, L., Pick, M., \& Démoulin, P. 1996, ApJ, 468, L73

Mikic, Z., \& Linker, J. A. 1994, ApJ, 430, 898

Mikic, Z., Schnack, D. D., \& van Hoven, G. 1990, ApJ, 361, 690

Pevtsov, A. A., \& Canfield, R. C. 1999, in Magnetic Helicity in Space and Laboratory Plasmas, Geophys. Monogr. 111, 103

Pevtsov, A. A., Canfield, R. C., \& Metcalf, T. R. 1995, ApJ, 440, L109

Régnier, S., Amari, T., \& Kersalé, E. 2002, A\&A, 392, 1119

Roumeliotis, G., Sturrock, P. A., \& Antiochos, S. K. 1994, ApJ, 423, 847

Rust, D. M., \& Kumar, A. 1996, ApJ, 464, L199

Schmieder, B., Démoulin, P., Aulanier, G., \& Golub, L. 1996, ApJ, 467, 881

Seehafer, N. 1990, Sol. Phys., 125, 219

Srivastava, N., Schwenn, R., Inhester, B., Martin, S. F., \& Hanaoka, Y. 2000, ApJ, 534, 468
Srivastava, N., Schwenn, R., \& Stenborg, G. 1999, in ESA SP-446: 8th SOHO Workshop: Plasma Dynamics and Diagnostics in the Solar Transition Region and Corona, 621

Sterling, A. C., \& Hudson, H. S. 1997, ApJ, 491, L55

Sturrock, P. A., Antiochos, S. K., \& Roumeliotis, G. 1995, ApJ, 443, 804

Török, T., \& Kliem, B. 2003, A\&A, 406, 1043

Tokman, M., \& Bellan, P. M. 2002, ApJ, 567, 1202

van Driel-Gesztelyi, L., Manoharan, P. K., Démoulin, P., et al. 2000, J. Atmospheric and Terrestrial Phys., 62, 1437

van Hoven, G., Mok, Y., \& Mikic, Z. 1995, ApJ, 440, L105

van Tend, W., \& Kuperus, M. 1978, Sol. Phys., 59, 115

Wang, H., Qiu, J., Jing, J., \& Zhang, H. 2003, ApJ, 593, 564

Zhang, J., Dere, K. P., Howard, R. A., \& Vourlidas, A. 2004, ApJ, 604, 420 